\title{
A Regularized Discrete Laminate Parametrization Technique with Applications to Wing-Box Design Optimization
}

\author{
Graeme J. Kennedy, ${ }^{*}$ \\ University of Toronto Institute for Aerospace Studies, Toronto, ON, Canada \\ Joaquim R. R. A. Martins ${ }^{\dagger}$ \\ University of Michigan, Department of Aerospace Engineering, Ann Arbor, MI, USA
}

\begin{abstract}
In this paper we present a novel ply parametrization technique for layered composites. This parametrization takes into account important manufacturing considerations, including the discrete selection of ply angles, the discrete nature of the layup thickness, and the continuity of ply angles across adjacent structural elements. The proposed technique can be used as either an alternative or as an enhancement to SIMP-type methods, such as discrete material optimization (DMO). We apply the proposed laminate parametrization technique to several structural optimization problems, including compliance minimization, failure-margin maximization, and buckling-load maximization, that have thousands of design variables and hundreds of constraints. These large design problems can only be solved, within a reasonable computational time, using gradient-based optimization methods with efficient sensitivity methods. Unlike mixed-integer approaches, our proposed laminate parametrization technique is well suited for gradient-based design optimization. As a final example, we apply the proposed parametrization method to the sizing and lamination sequence design of a composite wing-box. These results demonstrate that the proposed parametrization is effective for a wide range of structural optimization problems.
\end{abstract}

\section{Introduction}

The parametrization of composite structures for design optimization is a challenging problem. Realistic structural design optimization problems require numerous manufacturing constraints. Additionally, these design problems often involve a selection amongst a discrete set of options. For instance, the selection of ply angles from a set of available manufacturing options, or the selection of a laminated composite thickness that must be a integer multiple of the laminae thickness. These discrete problems are not, in their most natural form, amenable to gradient-based optimization. On the other hand, methods for nonlinear mixed-integer programming are almost inevitably computationally expensive, especially for large numbers of design variables. Here, we examine a laminate parametrization for structural design studies that takes into account the discrete nature of some of the design variables. In order to avoid solving a large, nonlinear, mixed-integer program, we use a relaxation approach where the original discrete problem is transformed into a continuous analogue of the original problem. We then obtain solutions to the modified problem using gradient-based optimization. To illustrate the effectiveness of the proposed laminate parametrization, especially for non-symmetric laminates, we examine a series of structural optimization problems of increasing difficulty.

Many authors have developed different laminate parametrization techniques. These generally fall into two categories: direct parametrizations that provide an explicit description of the physical laminate, and indirect parametrizations in which intermediate variables are employed and the lamination sequence is only available in a post-processing calculation. There are difficulties with using either of these approaches. Direct techniques often introduce many local minima in the design space, while indirect methods make it difficult to impose manufacturing constraints on the physical construction of the laminate. Here we use a direct parametrization of the laminate and accept the possibility of local minima.

The remainder of the paper is structured as follows. In Section II we review relevant literature on laminate parametrization techniques. In Section III we describe our proposed laminate parametrization technique and develop a method for predicting material failure within the context of the laminate parametrization. In Section IV we present an approach for discrete thickness selection. In Section V we present results from a series of structural optimization studies. Finally, in Section VI, we present an application of the proposed laminate parametrization technique to a wing-box sizing and layup-determination study.

\footnotetext{
*Ph.D. Candidate, AIAA Student Member

${ }^{\dagger}$ Associate Professor, AIAA Senior Member
} 


\section{Literature review}

Many authors have proposed laminate parametrization methods. In this section we review the most common methods, with a particular focus on techniques with similarities to our proposed approach.

Lamination parameters $[1,2]$, represent an implicit parametrization of the laminate stiffness with 12 lamination parameters and terms accounting for the constitutive properties of the lamina. Not all combinations of the lamination parameters represent physically realizable laminates so additional constraints must be imposed to restrict the domain of the laminates. The full feasible set is not known, so often a subset of the full parametrization is used. Lamination parameters have often been used as a parametrization for stiffness design $[3,4,5]$, and buckling design $[5,6]$. Other interesting uses of lamination parameters include avoiding local minima [7] and incorporating the Tsai-Wu [8] failure criteria into the lamination parameter space to obtain a conservative failure envelope [9]. The difficulty with the lamination parameter approaches is that they do not provide a direct description of the laminate construction. This makes it difficult to impose constraints on ply angles and thicknesses that may be required due to manufacturing considerations. In addition, the lamination parameter approach does not allow for a variation of the material type between layers.

The use of ply angles and number of plies is the most direct parametrization of a laminate, however this approach suffers from several problems. The laminate thickness is an important variable in preliminary design studies. However, if the same material is used for all layers, the thickness must be an integer multiple of the lamina ply thickness. As a result, this approach often results in a nonlinear mixed-integer program. Furthermore, for a fixed laminate thickness, with variable ply angles, the direct approach often introduces many local minima [10]. These properties make gradientbased design optimization challenging. Nevertheless, authors have reported various techniques to avoid these local optima [11, 12], or used gradient-free approaches [13].

Other authors have developed approaches that treat the discrete selection of ply angles and laminate thickness directly using analogies from topology optimization $[14,10,15,16]$. Discrete material optimization (DMO), with several variants on the proposed penalization, has achieved the most success. These approaches have often been limited to compliance design problems, however other interesting applications include design for minimum direct operational cost with manufacturing constraints [17]. The difficulty with DMO approaches is that they often fail to fully converge to a final design. It may be difficult to assess the merits of an intermediate solution.

\section{The proposed laminate parametrization}

The laminate parametrization described below is designed to implicitly satisfy manufacturing requirements that arise when parts of the structure are manufactured using hand laying techniques or automated tape laying. Here we assume that the wing structure is composed of wing skin, stiffeners, ribs and spars that are either bonded post-cure or co-cured into a complete assembly.

Keeping these manufacturing techniques in mind, we consider the following constraints on the laminate construction:

1. The ply angles must be selected from a discrete set of allowable angles, $\Theta=\left\{\theta_{1}, \theta_{2}, \ldots, \theta_{K}\right\}$.

2. The plies must be continuous over the manufactured component. Variations in the thickness are only possible by eliminating or adding plies to the existing layup.

3. The laminate thickness may be modified only by removing plies from the top of the layup, not from the bottom.

The intent of these constraints is to reflect manufacturing considerations that arise when using layered composite materials.

In the laminate parametrization technique, we consider a structure that is split into a series of $M$ design segments. Each design segment corresponds to a part of the structure with different properties than the immediately adjacent parts of the structure, subject to the constraints outlined above. Each design segment of the structure is modeled using first-order shear deformation theory (FSDT), where the in-plane, bending-stretching coupling, bending and transverse shear constitutive matrices are: $\mathbf{A}^{(i)}, \mathbf{B}^{(i)}, \mathbf{D}^{(i)}, \mathbf{A}_{s}^{(i)}$. Here the superscript $i$ indexes the $i$-th design segment, where $i=1, \ldots, M$.

In the following description, we first outline the proposed laminate parametrization using a mixed-integer formulation. We then proceed to relax the discrete problem to a continuous formulation. In the proposed parametrization technique, we express the constitutive matrices, $\mathbf{A}^{(i)}, \mathbf{B}^{(i)}, \mathbf{D}^{(i)}, \mathbf{A}_{s}^{(i)}$, in terms of a series of discrete ply-identity 
variables $\xi_{i j k} \in\{0,1\}$ :

$$
\begin{aligned}
\mathbf{A}^{(i)} & =\sum_{j=1}^{N}\left(h_{i j+1}-h_{i j}\right) \sum_{k=1}^{K} \xi_{i j k} \overline{\mathbf{Q}}\left(\theta_{k}\right), & \mathbf{B}^{(i)} & =\sum_{j=1}^{N} \frac{1}{2}\left(h_{i j+1}^{2}-h_{i j}^{2}\right) \sum_{k=1}^{K} \xi_{i j k} \overline{\mathbf{Q}}\left(\theta_{k}\right), \\
\mathbf{D}^{(i)} & =\sum_{j=1}^{N} \frac{1}{3}\left(h_{i j+1}^{3}-h_{i j}^{3}\right) \sum_{k=1}^{K} \xi_{i j k} \overline{\mathbf{Q}}\left(\theta_{k}\right), & \mathbf{A}_{\mathbf{s}}^{(i)} & =\kappa \sum_{j=1}^{N}\left(h_{i j+1}-h_{i j}\right) \sum_{k=1}^{K} \xi_{i j k} \overline{\mathbf{Q}}_{s}\left(\theta_{k}\right),
\end{aligned}
$$

where there are $N$ plies in the laminate, $\overline{\mathbf{Q}}(\theta)$ and $\overline{\mathbf{Q}}_{s}(\theta)$ are the laminae in-plane and shear stiffnesses in the global coordinate system [8], and $h_{i j}$ are the through-thickness coordinate of the $j$-th layer-interface in the $i$-th design segment. In this presentation we will fix $N$ for all design segments to simplify the presentation. In general, the number of plies may vary between design segments.

An active ply-identity variable $\xi_{i j k}=1$, indicates that the $k$-th ply angle, $\theta_{k}$, in the $j$-th layer of the $i$-th design segment has been selected. To avoid selecting multiple ply angles in the same layer we impose the following constraint:

$$
\sum_{k=1}^{K} \xi_{i j k}=1, \quad i=1, \ldots, N, \quad j=1, \ldots, M .
$$

This discrete formulation is identical to the linear mixed-integer approach of Haftka and Walsh [18]. Equation (2) ensures that one and only one ply is active in each layer, thus $\xi_{i j p}=1$ for some $p$, while $\xi_{i j k}=0$ for $k \neq p$.

The number of possible designs increases rapidly as the number of ply angles, layers and design segments increase. Evaluating all possible designs quickly becomes computationally intractable as there are $(M N)^{K}$ possible combinations.

Instead of using the discrete variables $\xi_{i j k} \in\{0,1\}$, we relax the mixed-integer problem and use continuous variables, which we write as: $x_{i j k} \in[0,1]$. We refer to continuous designs that satisfy $x_{i j k} \in\{0,1\}$ as $0-1$ solutions. The stiffness can now be expressed in terms of the continuous ply-identity variables as follows:

$$
\begin{aligned}
\mathbf{A}^{(i)} & =\sum_{j=1}^{N}\left(h_{i j+1}-h_{i j}\right) \sum_{k=1}^{K} x_{i j k}^{P} \overline{\mathbf{Q}}\left(\theta_{k}\right), & \mathbf{B}^{(i)} & =\sum_{j=1}^{N} \frac{1}{2}\left(h_{i j+1}^{2}-h_{i j}^{2}\right) \sum_{k=1}^{K} x_{i j k}^{P} \overline{\mathbf{Q}}\left(\theta_{k}\right), \\
\mathbf{D}^{(i)} & =\sum_{j=1}^{N} \frac{1}{3}\left(h_{i j+1}^{3}-h_{i j}^{3}\right) \sum_{k=1}^{K} x_{i j k}^{P} \overline{\mathbf{Q}}\left(\theta_{k}\right), & \mathbf{A}_{\mathbf{s}}{ }^{(i)} & =\kappa \sum_{j=1}^{N}\left(h_{i j+1}-h_{i j}\right) \sum_{k=1}^{K} x_{i j k}^{P} \overline{\mathbf{Q}}_{s}\left(\theta_{k}\right),
\end{aligned}
$$

where $x_{i j k}$ are continuous over the interval $[0,1]$. Note that we have introduced a SIMP penalty parameter $P$ as an exponent on the continuous ply identity variables. The purpose of the parameter $P$ is to penalize the stiffness of intermediate designs such that $0-1$ points have more favorable stiffness-to-weight ratios. However, a $0-1$ solution is not guaranteed in general when using SIMP penalization alone, even for large values of the parameter $P[19,20]$.

As in the mixed-integer formulation, we impose the following constraint on the continuous ply-angle selection variables:

$$
\sum_{k=1}^{K} x_{i j k}=1, \quad i=1, \ldots, M, \quad j=1, \ldots, N .
$$

This constraint imposes that the weights are a partition of unity and ensures a reasonable interpolation of the material properties. In the discrete case this constraint is sufficient to ensure that a single material is active. However, in the continuous case, this constraint only forces the design variables to remain on a plane intersecting the coordinate axes at unity.

In the design problem we collect all the design variables into the design vector $\mathbf{x} \in \mathbb{R}^{M N K}$. We collect all the linear constraints (4) for each design patch and each layer into the following expression:

$$
\mathbf{A}_{w} \mathbf{x}=\mathbf{e}
$$

where $\mathbf{A}_{w} \in \mathbb{R}^{M N \times M N K}$ is a matrix and all the entries in the vector $\mathbf{e} \in \mathbb{R}^{M N}$ are unity.

In DMO and classical SIMP methods, the purpose of the penalization parameter $P$ is to push the design towards a $0-1$ by penalizing designs with intermediate densities. Often, a continuation approach is employed where a series of optimization problems are solved for increasing values of $P[21,22]$. In this paper we augment the SIMP approach 
with an exact penalization technique. In order to force the design towards a $0-1$ solution, we introduce the following additional constraint:

$$
\sum_{k=1}^{K} x_{i j k}^{2}=1, \quad i=1, \ldots, M, \quad j=1, \ldots, N .
$$

The conditions that the design variables remain on the interval $x_{i j k} \in[0,1]$, sum to unity, and remain on the unit $(K-1)$-sphere, are sufficient to ensure that only one $x_{i j k}$ is equal to unity for each layer. In fact, the upper limit on the design variables $x_{i j k}$ is redundant and may be dropped. These criteria are shown graphically in Figure (1) for $K=3$ as the intersection of a 2 -sphere and a plane for $x_{1}, x_{2}, x_{3} \geq 0$.

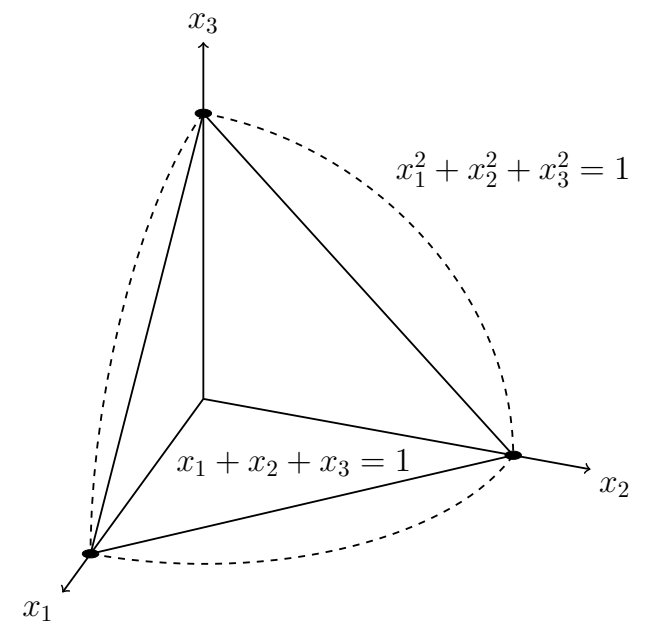

Figure 1: An illustration of the spherical constraint, forcing a selection of a single ply angle selection for each layer. This generalizes to arbitrary dimensions beyond $K=3$.

For ease of presentation, the spherical constraints for all layers in all design segments are collected into a single vector constraint, written as follows:

$$
\mathbf{c}_{s}(\mathbf{x})-\mathbf{e}=0,
$$

where $\mathbf{c}_{s}(\mathbf{x}) \in \mathbb{R}^{M N}$ and $\mathbf{e} \in \mathbb{R}^{M N}$.

If the objective of interest is $f(\mathbf{x})$, and any additional design constraints are written as $\mathbf{h}(\mathbf{x}) \geq 0$, the design optimization problem, with the constraints (5) and (7), is:

$$
\begin{aligned}
\operatorname{minimize} & f(\mathbf{x}) \\
\text { w.r.t. } & \mathbf{x} \geq 0 \\
\text { s.t. } & \mathbf{h}(\mathbf{x}) \geq 0 \\
& \mathbf{c}_{s}(\mathbf{x})-\mathbf{e}=0 \\
& \mathbf{A}_{w} \mathbf{x}-\mathbf{e}=0
\end{aligned}
$$

The difficulty with this problem is that the spherical constraints (7) are highly nonlinear and introduce many local minima. In order to control this effect, we relax the spherical constraint (7) and introduce it through an $\ell_{1}$ penalty function with penalty parameter $\gamma$. The objective of this modified problem is $f(\mathbf{x})+\gamma\left\|\mathbf{c}_{s}(\mathbf{x})-\mathbf{e}\right\|_{1}$ where $\|\cdot\|_{1}$ is the $\ell_{1}$ norm. However, this modified objective is not differentiable. Instead, we use an elastic programming technique [23], that creates the effect of the $\ell_{1}$ norm in a differentiable manner by adding additional slack variables to the optimization problem. Using the elastic programming approach, we introduce the vectors of slack variables $\mathbf{s}_{+}, \mathbf{s}_{-} \in \mathbb{R}^{M N}$ such that

$$
\mathbf{c}_{s}(\mathbf{x})-\mathbf{e}=\mathbf{s}_{+}-\mathbf{s}_{-},
$$

where we impose $\mathbf{s}_{+}, \mathbf{s}_{-} \geq 0$. The slack variables $\mathbf{s}_{+}$and $\mathbf{s}_{-}$, represent the positive and negative constraint violation of Equation (7). 
The modified optimization problem becomes:

$$
\begin{aligned}
\operatorname{minimize} & f(\mathbf{x})+\gamma \mathbf{e}^{T}\left(\mathbf{s}_{+}+\mathbf{s}_{-}\right) \\
\text {w.r.t. } & \mathbf{x}, \mathbf{s}_{+}, \mathbf{s}_{-} \geq 0 \\
\text { s.t. } & \mathbf{h}(\mathbf{x}) \geq 0 \\
& \mathbf{c}_{s}(\mathbf{x})-\mathbf{e}=\mathbf{s}_{+}-\mathbf{s}_{-} \\
& \mathbf{A}_{w} \mathbf{x}-\mathbf{e}=0
\end{aligned}
$$

where the parameter $\gamma>0$ is a penalty parameter. For a feasible problem, with a sufficiently large, but finite value of $\gamma$, Problem (10) admits a solution $\mathbf{x}^{*}, \mathbf{s}_{+}^{*}=\mathbf{s}_{-}^{*}=0$, that is also a solution to Problem (8). However, as $\gamma \rightarrow 0$, the problem admits other solutions that do not satisfy the $0-1$ criteria. Our approach will be to solve the optimization problem (10) for increasing values of penalty parameter. Initially, this will allow greater freedom in exploring the design space, but with increasing $\gamma$, the solution will tend either towards local minima or a 0-1 solution.

A further simplification of Problem (10) can be achieved when the summation constraints (5) are satisfied exactly. Starting from Equation (5), the sum of the squared design variables must be less than one, i.e.

$$
1=\left(\sum_{k=1}^{K} x_{i j k}\right)^{2} \geq \sum_{k=1}^{K} x_{i j k}^{2}
$$

As a result, when the linear constraint (5) is satisfied exactly, the constraint violation of Equation (7) is negative, i.e. $\mathbf{c}_{s}(\mathbf{x})-\mathbf{e} \leq 0$. Therefore, the values of the slacks at the solution are:

$$
\begin{aligned}
& \mathbf{s}_{+}^{*}=0, \\
& \mathbf{s}_{-}^{*}=\mathbf{e}-\mathbf{c}_{s}\left(\mathbf{x}^{*}\right) .
\end{aligned}
$$

This result can also be observed geometrically. Whenever the design lies on the plane, the normal distance from the plane to the sphere is strictly positive, unless it is at a 0-1 point when the normal distance is precisely zero, see Figure 1.

If the linear constraints are satisfied at all iterations, the optimization problem can be further simplified. We denote this optimization problem $\operatorname{Opt}(\gamma)$, written as follows:

$$
\begin{aligned}
\operatorname{minimize} & f(\mathbf{x})+\gamma \mathbf{e}^{T}\left(\mathbf{e}-\mathbf{c}_{s}(\mathbf{x})\right) \\
\text { w.r.t. } & \mathbf{x} \geq 0 \\
\text { s.t. } & \mathbf{h}(\mathbf{x}) \geq 0 \\
& \mathbf{A}_{w} \mathbf{x} \equiv \mathbf{e}
\end{aligned}
$$

where the final constraint is written as $\mathbf{A}_{w} \mathbf{x} \equiv \mathbf{e}$ to indicate that it is satisfied at every iteration.

We use a continuation approach and solve $\operatorname{Opt}\left(\gamma_{n}\right)$ for a sequence of increasing $\gamma_{n}$, starting each subsequent optimization problem from the previous solution. In this paper, we use the sequential quadratic optimization code SNOPT [24], through the Python-based wrapper in the optimization package pyOpt [25]. SNOPT implicitly satisfies the linear constraints exactly at every iteration.

\section{A. Failure prediction}

The prediction of structural failure is a critical aspect of preliminary structural sizing. Here we propose an extension of the parametrization technique presented above that provides a failure envelope for each layer of the laminate. We use the generic failure criteria

$$
F(\boldsymbol{\sigma}) \leq 1
$$

In the remainder of this paper, we use the Tsai-Wu failure criterion exclusively. In practice, any failure criterion that takes the form of Equation (12) could be used.

The application of the failure criterion poses no problems at $0-1$ points. The difficulty is that it is also necessary to predict failure at intermediate designs. In order to overcome this issue, we interpolate the failure criterion such that the full criterion is applied at 0-1 points and a reasonable criterion is employed at intermediate designs. We use following interpolation strategy:

$$
F^{(i j)}(\boldsymbol{\sigma})=\sum_{k} \psi\left(x_{i j k}\right) F\left(\boldsymbol{\sigma}^{(k)}\right), \quad i=1, \ldots, M, \quad j=1, \ldots, M
$$


where $F^{(i j)}$ is the value of the interpolated criterion for each design segment $i$ for every layer $j, \sigma^{(k)}$ is the stress in the material axis for the $k$-th ply angle, and $\psi(\cdot)$ is an interpolation function that decreases the failure margin artificially at intermediate designs. Following previous work on topology optimization [26], we use the function

$$
\psi(s)=\frac{s}{\epsilon(1-s)+s},
$$

where typically $\epsilon=0.3$. This interpolation increases the failure criterion artificially at intermediate values. An important property of this interpolation function is that its derivative takes finite values over the entire interval. Other interpolation strategies used in SIMP methods such as $\psi(s)=s^{1 / p}$ for $p>1$ do not share this property and could not be used here.

\section{B. Adjacency constraints}

In this section, we introduce an additional restriction on the design variables such that the active ply may only shift a specified number of positions within the set $\Theta$. This type of constraint could arise in a number of situations. In the case of laminate design, it may be necessary to impose a constraint to prevent large changes in the ply angles between adjacent segments, or between layers, due to manufacturing or design considerations. The implementation of this type of adjacency constraint is described below.

Without loss of generality, consider first layer $j=1$ of the design segments $i=1$ and $i=2$ with design variables $x_{11 k}$ and $x_{21 p}$ respectively. If the design variable $x_{11 k}$ is active at the solution, then the purpose of the adjacency constraint is to restrict available choices in the next design segment to the closest $L$ options in the range $x_{21(k-L)}, \ldots, x_{21(k+L)}$. As a result the design variables are restricted from shifting more than $L$ positions between segments.

This type of constraint can be imposed as follows:

$$
\begin{aligned}
x_{11 k} x_{21 p} \leq 0, & k \in\{1,2, \ldots, K\}, \\
& p \in I_{k},
\end{aligned}
$$

where $I_{k}$ is a set of indices that contains the adjacent design variables that cannot be used. This type of constraint, in combination with the condition $x_{i j k} \geq 0$, is known as a complementarity constraint [23]. The less-than condition is used to conform to the standard complementarity constraint notation. Unfortunately, this type of constraint violates conventional constraint qualifications such as the Mangasarian-Fromovitz constraint qualification (MFCQ) or the linear independence constraint qualification (LICQ) [23]. This constraint qualification failure can be observed by noting that the constraint gradients of Equation (14) are zero when the constraint is active. As a result, these types of constraints do not admit Lagrange multipliers at the solution, and gradient-based optimizers may encounter difficulties approaching the solution $[27,28]$.

Instead of trying to use the complementarity constraint (14) directly, we use a regularization of the constraint due to Scholtes [28]. In this regularization technique, the original constraint is perturbed in the following manner:

$$
\begin{aligned}
x_{11 k} x_{21 p} \leq \tau, & k \in\{1,2, \ldots, N\}, \\
& p \in I_{k},
\end{aligned}
$$

for $\tau>0$. Note that the gradients of this constraint are non-zero on the boundary, $x_{11 k} x_{21 p}=\tau$. In the approach of Scholtes [28], a series of optimization problems are then solved for decreasing values of $\tau$ using a conventional SQPbased optimizer, starting each new problem from the previous solution. This series of perturbed problems converges to a solution of the original problem with some conditions on the linear independence of the constraint gradients excluding the complementarity constraint (14) [28].

Here we consider two options for the set of indices $I_{k}$. In the first case, the index set is constructed so that design variable in one segments may only take on immediately adjacent designs in the second segment. In this case, the index set is defined as follows,

$$
I_{k}=\{1,2, \ldots, K\} \backslash\{k-L, \ldots, k+L\} .
$$

In the second case, the design selections are allowed to wrap around. For instance if the design variable $x_{111}$ is active, then $x_{211}$ through $x_{21 L}$, as well as, $x_{21 N}$ through $x_{21(N-L)}$ could be active as well. In this case the index set is defined as:

$$
I_{k}=\left\{\begin{array}{ll}
k \leq L & \{k+L+1, \ldots, K+k-L-1\} \\
k \geq K-L & \{k-K+L+1, \ldots, k-L-1\} \\
\text { otherwise } & \{1,2, \ldots, K\} \backslash\{k-L, \ldots, k+L\}
\end{array} .\right.
$$


For concreteness, consider the case when $\Theta=\left\{-45^{\circ}, 0^{\circ}, 45^{\circ}, 90^{\circ}\right\}$ with $L=1$. In this case, the first group of sets $I_{k}$ from Equation (16) would be:

$$
\begin{aligned}
& I_{1}=\{3,4\}, \quad I_{2}=\{4\}, \quad I_{3}=\{1\}, \quad I_{4}=\{1,2\}, \\
& \Theta\left(I_{1}\right)=\left\{45^{\circ}, 90^{\circ}\right\}, \quad \Theta\left(I_{2}\right)=\left\{90^{\circ}\right\}, \quad \Theta\left(I_{3}\right)=\left\{-45^{\circ}\right\}, \quad \Theta\left(I_{4}\right)=\left\{-45^{\circ}, 0^{\circ}\right\} .
\end{aligned}
$$

The second case from Equation (17), with wrap around, would be:

$$
\begin{aligned}
& I_{1}=\{3\}, \quad I_{2}=\{4\}, \quad I_{3}=\{1\}, \quad I_{4}=\{2\}, \\
& \Theta\left(I_{1}\right)=\left\{45^{\circ}\right\}, \quad \Theta\left(I_{2}\right)=\left\{90^{\circ}\right\}, \quad \Theta\left(I_{3}\right)=\left\{-45^{\circ}\right\}, \quad \Theta\left(I_{4}\right)=\left\{0^{\circ}\right\} .
\end{aligned}
$$

Note that these sets represent the adjacent angles that are not permitted, given the active angle in the current layer.

The total number of adjacency constraints can be reduced by combining groups of the constraints (14) into a single equivalent constraint. Here, we use the equivalent constraint:

$$
x_{11 k} \sum_{p \in I_{k}} x_{21 p} \leq \tau, \quad k \in\{1,2, \ldots, K\} .
$$

A further reduction could be made by adding these constraints together for all $k$.

For ease of presentation, we write all of the grouped adjacency constraints (18) in the following form:

$$
\mathbf{d}(\mathbf{x}) \leq \tau,
$$

where $\mathbf{d} \in \mathbb{R}^{n_{a}}$, where $n_{a}$ is the number of adjacency constraints in the form of Equation (18).

The original optimization problem (11) with the additional adjacency constraints as $\operatorname{Opt}^{\prime}(\gamma, \tau)$, can be written as follows:

$$
\begin{aligned}
\min & f(\mathbf{x})+\gamma \mathbf{e}^{T}\left(\mathbf{e}-\mathbf{c}_{s}(\mathbf{x})\right) \\
\text { w.r.t. } & \mathbf{x} \geq 0 \\
\text { s.t. } & \mathbf{h}(\mathbf{x}) \geq 0 \\
& \mathbf{d}(\mathbf{x}) \leq \tau \\
& \mathbf{A}_{w} \mathbf{x} \equiv \mathbf{e}
\end{aligned}
$$

Here we solve the optimization problem $\operatorname{Opt}^{\prime}\left(\gamma_{n}, \tau_{n}\right)$ for a series $\left\{\gamma_{n}, \tau_{n}\right\}$, with non-decreasing $\gamma_{n}$ and non-increasing $\tau_{n}$.

\section{Structural thickness selection}

The discrete material parametrization approach outlined above can be extended to problems in which the thickness of the laminate is also a discrete variable. We have implemented such an approach and have found that it suffers from several issues that are not often present in ply angle selection problems.

To illustrate these issues, consider the stiffness-to-weight maximization of an isotropic plate where the thickness is limited to three discrete values. In the continuation approach, the penalty parameter is incremented after each optimization. When no penalty is applied, it is advantageous to select a portion of the thickness and thinnest selections as this intermediate material often provides the highest stiffness-to-weight ratio. However, as the penalization is incremented, the intermediate material may be the best selection, but the design obtained from the initial, optimization without penalization will have zero contribution from the intermediate thickness. As a result, the continuation approach does not work well in this case because the initial optimization does not bring the solution closer to the final result.

This example illustrates that continuation methods rely on the assumption that the intermediate penalized solution is close to a discrete solution, where the "closeness" of the solution is measured in the design space. Within the context of this example, although the weighted-average thickness may be close to a discrete thickness value, the design weights themselves may be far from the discrete solution.

In order to address this issue, we have developed a discrete optimization approach for thickness variables. This approach can be applied to any problem in which the stiffness and mass are continuous, monotonically increasing functions of the thickness. In particular, this approach can be used for isotropic plates and composite laminates where lamination parameters are employed. With this restriction, the discrete thickness approach is not as flexible as the discrete material selection approach outlined above. However, we have found that it is well suited for problems in which the thickness must be selected from a discrete set of values. 


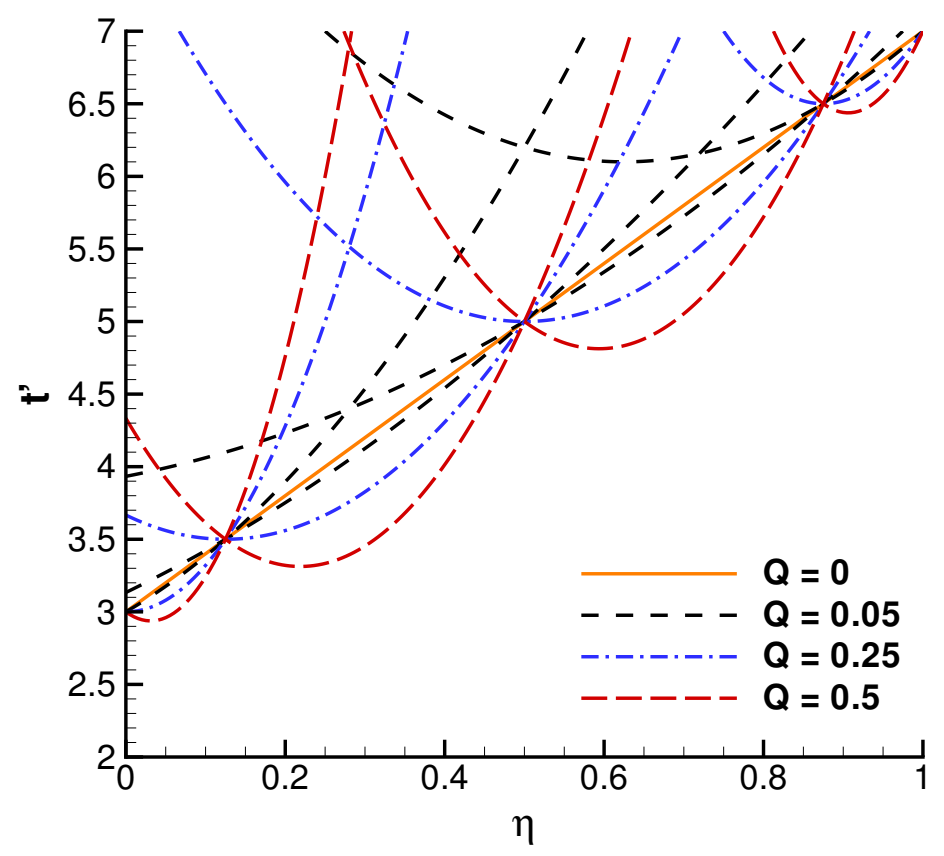

Figure 2: The discrete thickness constraint for increasing values of the penalty parameter $Q$, for discrete thicknesses $3,3.5,5,6.5$ and $7 \mathrm{~mm}$.

\section{A. The discrete thickness selection problem}

We introduce two design variables in the thickness selection problem: a constrained thickness variable, $t^{\prime}$, and an interpolation variable, $\eta$. The constrained thickness $t^{\prime}$ is used to penalize intermediate values of the thickness, while $\eta$ is used to interpolate the constraint values.

The goal of the discrete thickness parametrization is to force $t^{\prime}$ towards a value in the ordered set of discrete thicknesses: $\mathcal{T}=\left\{t_{1}, t_{2}, \ldots, t_{N}\right\}$, where the members of the set $\mathcal{T}$ are ordered such that $t_{k+1}>t_{k}$.

In order to force the solution towards members of the set $\mathcal{T}$, we impose a series of constraints such that intermediate solutions have lower stiffness-to-weight and strength-to-weight ratios. This is identical to the approach taken in SIMP methods. However, a SIMP-type approach to penalization cannot be used directly because there may be more than two thicknesses. Furthermore, any penalization approach must be differentiable at the discrete thicknesses.

To retain a differentiable approach we enforce an effective penalization using a series of constraints. Given the set of discrete thicknesses $\mathcal{T}$, we impose the following set of $N$ constraints:

$$
\begin{aligned}
t^{\prime}-t_{1}-\left(t_{N}-t_{1}\right) \eta & \leq 0, \\
t^{\prime}-\left(t_{k}(1-u)+t_{k+1} u-4 Q\left(t_{k+1}-t_{k}\right)\left(u-u^{2}\right)\right) & \leq 0, \quad k=1, \ldots, N-1,
\end{aligned}
$$

where $\eta_{k}$ are the parametric points $\eta_{k}=\left(t_{k}-t_{1}\right) /\left(t_{N}-t_{1}\right), u$ is an interpolation variable defined as $u=(\eta-$ $\left.\eta_{k}\right) /\left(\eta_{k+1}-\eta_{k}\right)$, and $Q$ represents a penalization parameter. The constraints (21) are imposed such that the quadratic passes through $t_{k}, t_{k+1}$ at $u=0$ and $u=1$ respectively, as well as the point $u=1 / 2,\left(t_{k}+t_{k+1}\right) / 2-Q\left(t_{k+1}-t_{k}\right)$. Thus, $Q$ represents the penalization as a fraction of the increment $t_{k+1}-t_{k}$.

When computing the mass and strength properties of the laminate, we use the interpolated thickness $t(\eta)=$ $t_{1}+\left(t_{N}-t_{1}\right) \eta$, while for the stiffness properties, we use the constrained thickness $t^{\prime}$. At feasible points $t^{\prime}<t(\eta)$, and a result, intermediate thickness values have lower stiffness-to-weight and strength-to-weight ratios. In fact, for sufficiently large $Q$, each discrete thickness $t^{\prime}=t \in \mathcal{T}$ is a local maximum of the stiffness-to-weight ratio.

For ease of presentation, we group all the design variables $t^{\prime}$ and $\eta$ into the vector of design variables $\mathbf{t}$ and group the constraints from Equation (21) into a vector of constraints $\mathbf{g}(\mathbf{t}) \leq 0$ such that the design problem $\operatorname{DOpt}(Q)$ can be 
written as follows:

$$
\begin{aligned}
\operatorname{minimize} & f(\mathbf{t}) \\
\text { w.r.t. } & \mathbf{t} \\
\text { s.t. } & \mathbf{h}(\mathbf{t}) \geq 0 \\
& \mathbf{g}(\mathbf{t}) \leq 0
\end{aligned}
$$

Here we solve the problem $\operatorname{DOpt}\left(Q_{n}\right)$ for a series of increasing values of $Q_{n}$, starting each new optimization problem from the solution to the previous problem.

Figure 2 shows the set of constraints (21) for the set of thicknesses $\mathcal{T}=\{3,3.5,5,6.5,7\}$ for increasing values of the penalty parameter $Q=0,0.05,0.25$ and 0.5 . Note that the feasible space of designs are those values of $t^{\prime}$ less than the lowest penalization curve and less than the interpolated value of $t(\eta)$.

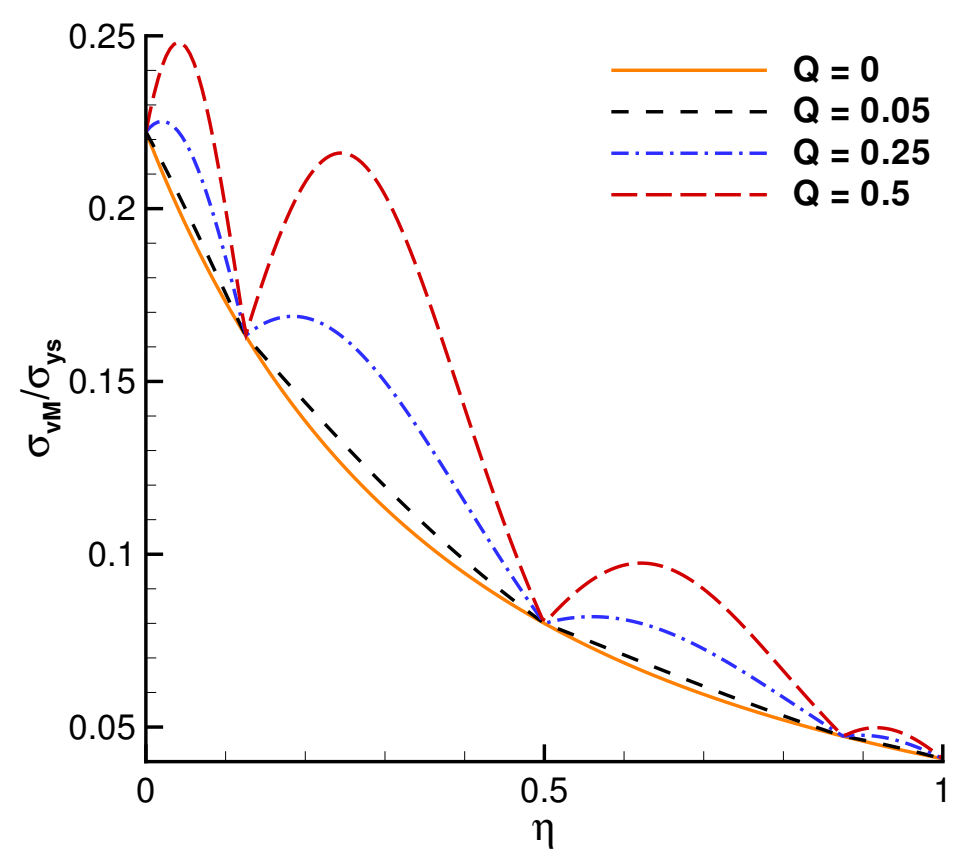

Figure 3: The trace of the normalized von Mises stress along the feasible domain as a function of $\eta$ for discrete thicknesses $3,3.5,5,6.5$ and $7 \mathrm{~mm}$, with increasing values of the penalty parameter $Q$. The plot shows the penalization of the intermediate designs.

The discrete thickness approach can also be applied to strength-based optimization problems. Figure 3 shows the ratio of the von Mises stress to the yield stress for a constant applied bending moment for the set of thicknesses $\mathcal{T}=$ $\{3,3.5,5,6.5,7\}$ with increasing values of the penalty parameter $Q=0,0.05,0.25$ and 0.5 . Here we have plotted the trace of the feasible space: at each $\eta$ we find the maximum $t^{\prime}$ that is feasible with respect to the constraints (21). It is clear that the discrete thickness values provide the best strength-to-weight ratios at sufficiently large values of $Q$. Note that the distribution in Figure 3 appears to be non-differentiable. The apparent non-differentiable points are due to a switch between constraints defined in Equation (21). The constraints are smooth, within the context of the optimization problem itself.

In this section, we presented a penalization-based approach for discrete thickness selection problems. There are several differences between this discrete thickness approach and the discrete material optimization approach presented in Section III:

- The discrete thickness approach is an approximate penalization based on a physical observation: penalization of the stiffness-to-weight and strength-to-weight ratio, in many cases, pushes the design towards a discrete solution. This, however, is not guaranteed. Within the discrete material selection approach, the penalization is exact in the sense that the $0-1$ points will be local minima of the optimization problem for sufficiently large values of the penalty parameter $\gamma$. 
- The discrete thickness approach requires fewer variables than adding identity variables for each thickness parameter. However, many more constraints are required: one constraint for each discrete thickness.

\section{Structural optimization studies}

We now present a series of structural optimization problems that demonstrate both the laminate parametrization method and the discrete thickness selection approach. These examples involve both isotropic and composite materials. The representative isotropic and composite material properties used for all examples in this section are listed in Table 1.

\begin{tabular}{|c|c|c|c|c|c|c|c|}
\hline \multicolumn{2}{|c|}{ Stiffness [GPa] } & \multicolumn{2}{|c|}{ Poisson's ratio } & \multicolumn{2}{|c|}{ Strength [MPa] } & \multicolumn{2}{|c|}{ Density $\left[\mathrm{kg} / \mathrm{m}^{3}\right]$} \\
\hline \multicolumn{8}{|c|}{ Aluminum material properties } \\
\hline$E$ & 70.0 & $\nu$ & 0.3 & $\sigma_{y s}$ & 343.0 & $\rho$ & 2780 \\
\hline \multicolumn{8}{|c|}{ Composite material properties } \\
\hline$E_{11}$ & 164.0 & $\nu_{12}$ & 0.34 & $X_{t}$ & 2410 & $\rho$ & 1580 \\
\hline$E_{22}$ & 8.3 & & & $X_{c}$ & 1040 & & \\
\hline$G_{12}$ & 21.0 & & & $Y_{t}$ & 73.0 & & \\
\hline$G_{13}$ & 21.0 & & & $Y_{c}$ & 173.0 & & \\
\hline$G_{23}$ & 12.0 & & & $S$ & 183.0 & & \\
\hline \multicolumn{8}{|c|}{ Ply thickness $t_{p}=0.125 \mathrm{~mm}$} \\
\hline
\end{tabular}

Table 1: Representative Aluminum and IM7/3501-6 stiffness and strength properties.

This section is organized as follows: First we present results that demonstrate the effectiveness of the discrete material selection approach for laminate parametrization. In Section A and Section B we present results for compcliance and failure-based optimization of a square composite plate, respectively. In both these examples we compare results with and without the adjacent ply constraints (19). Next, we present results that demonstrate the effectiveness of the discrete thickness selection approach. In Section C and Section D, we consider the compliance minimization and von Mises stress minimization of a clamped plate subject to a pressure load. In both cases we compare the discrete optimization results with an equivalent continuous optimization study. Finally, in Section E we present results of a critical buckling load maximization problem. In all cases, the finite-element analysis is performed using the Toolkit for the Analysis of Composite Structures (TACS) [29], an advanced parallel finite-element code with sophisticated sensitivity analysis capability. The efficient sensitivity analysis capabilities in TACS are extremely important for all the cases presented here, since large numbers of design variables are required for the laminate parametrization.

In these examples we have chosen bending-dominated structures rather than structures primarily loaded in the plane. Stacking sequence optimization for in-plane loadings can lead to non-unique design problems since the stacking sequence of a laminate does not modify the in-plane stiffness or strength properties. These bending-dominated structures present a challenging set of problems that demonstrate the wide range of applicability of both the discrete material selection and discrete thickness techniques presented in this paper.

\section{A. Compliance minimization of a square composite plate}

In this section, we consider the compliance minimization of a fully clamped plate that is subjected to a uniform surface pressure. The number of layers in the laminated plate is fixed at 8 and the laminate is parametrized with the lamination parametrization technique discussed in Section III. The plate is sub-divided into $9 \times 9$ design segments, each with 8 plies. Here, we restrict the lamination angles to $0^{\circ}, \pm 45^{\circ}$ and $90^{\circ}$, resulting in 4 ply identity variables per layer. As a result, there are 2592 ply identity design variables with 648 linear constrains.

The plate is $900 \times 900 \mathrm{~mm}$ and is subjected to a $1 \mathrm{kPa}$ pressure load, positive out of the page. This puts the bottom layer of the laminate in compression and the top layer of the laminate in tension at the middle of the plate. Each of the $9 \times 9$ design patches are modeled using $3 \times 3$, 3rd order MITC9 shell elements. The finite-element model contains 725 elements, 3025 nodes and just over 18000 structural degrees of freedom.

We solve this problem with and without the adjacency constraints introduced in Section B. Here we use the second formulation of the adjacency constraints (17) and apply the constraints for each ply between adjacent design segments along the coordinate directions, but not along the diagonal. As a result the ply angles are only permitted to change by $45^{\circ}$ between adjacent design segments. 
Here, we use the following optimization formulation which we denote $\operatorname{CompOpt}(P, \gamma, \tau)$ :

$$
\begin{aligned}
\operatorname{minimize} & \alpha \frac{1}{2} \mathbf{u}^{T} \mathbf{K u}+\gamma \mathbf{e}^{T}\left(\mathbf{e}-\mathbf{c}_{s}(\mathbf{x})\right) \\
\text { w.r.t. } & \mathbf{x} \geq 0 \\
\text { s.t. } & \mathbf{K u}=\mathbf{f} \\
& \mathbf{d}(x) \leq \tau \\
& \mathbf{A}_{w} \mathbf{x} \equiv \mathbf{e}
\end{aligned}
$$

where $\mathbf{K}$ is the stiffness matrix, $\mathbf{f}$ is the consistent force vector and $\alpha$ is a scaling parameter which we set to $\alpha=$ $1 / 20000$.

We solve a sequence of $\operatorname{problems} \operatorname{CompOpt}\left(P_{n}, \gamma_{n}, \tau_{n}\right)$, starting each new problem from the solution of the previous iteration. We solve the problem without any SIMP penalization and therefore set $P_{n}=1$ for all continuation iterations. For the first iteration we select the penalty parameter $\gamma_{1}=0$, and for all subsequent iterations we take $\gamma_{n}=10^{n-6}$ for $n \geq 2$. For the adjacency constraints, we use the sequence $\tau_{n}=(1 / 2)^{n}$. In all optimization problems we employ an optimality and feasibility tolerance $10^{-6}$.

Table 2 lists the results of the compliance minimization study with and without the constraints on adjacent ply angles. Note that in this table, the infeasibility is the constraint violation of the spherical constraint (6), measured using the $\ell_{1}$ norm. Both compliance minimization problems converge rapidly to a $0-1$ solution. The compliance minimization problem requires only two continuation iterations, whereas the compliance minimization with adjacency constraints requires an extra continuation step. It is clear that the first optimization solution is the most costly, while subsequent optimizations require fewer iterations.

Figure 4 shows the optimal ply angles for the compliance minimization problem, while Figure 5. Note that the composite plies are ordered from the bottom to the top of the laminate. Both solutions share some similar characteristics, especially at the middle of the plate in the outer-most plies. These plies have the largest contribution to the objective. The inner-plies of the adjacency-constrained result differ significantly from the unconstrained result.

\begin{tabular}{lccccccc}
\hline Iteration $P$ & $\gamma$ & $\tau$ & Compliance & Infeasibility & $\begin{array}{c}\text { Function } \\
\text { evaluations }\end{array}$ & $\begin{array}{l}\text { Gradient } \\
\text { evaluations }\end{array}$ \\
\hline \multicolumn{2}{l}{ No adjacency constraints } \\
\hline 1 & 1 & 0 & - & 19862.33 & 5.99 & 314 & 65 \\
2 & 1 & $10^{-3}$ & - & 19861.40 & $3.57 \times 10^{-11}$ & 36 & 14 \\
\hline \multicolumn{7}{l}{ With adjacency constraints } \\
\hline 1 & 1 & 0 & 0.5 & 21309.27 & 36.4 & 586 & 105 \\
2 & 1 & $10^{-3}$ & 0.25 & 20888.16 & 9.0 & 180 & 36 \\
3 & 1 & $10^{-2}$ & 0.125 & 20433.89 & $2.47 \times 10^{-13}$ & 40 & 12 \\
\hline
\end{tabular}

Table 2: Comparison between the compliance minimization results with and without adjacency constraints. Note that the infeasibility is calculated as $\left\|\mathbf{c}_{s}\left(\mathbf{x}_{n}^{*}\right)-\mathbf{e}\right\|_{1}$.

\section{B. Failure load maximization of a square composite plate}

In this section, we present results for a square clamped plate loaded with two separate constant pressure loads $\pm \lambda$. The plate is composed of 6 layers and is sub-divided into 25 design segments. Ply identity variables are employed for the angles $0^{\circ}, \pm 45^{\circ}$ and $90^{\circ}$, resulting in a total of 600 design variables.

The finite-element model of the plate consists of a $4 \times 4,3^{\text {rd }}$ order MITC9 shell elements for each design segment. The finite-element mesh consists of 400 elements, 1681 nodes and approximately 10000 degrees of freedom.

Here, we employ the Tsai-Wu failure criterion at each Gauss point with the elements of a single design segment. Instead of using the maximum value of the Tsai-Wu failure criterion over each design segment, we use a KreisselmeierSteinhauser (KS) aggregation technique to obtain an upper bound on the maximum value [30]. The optimization 


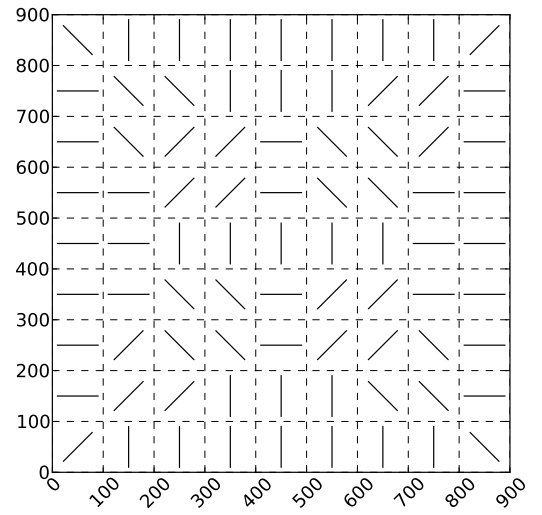

(a) Ply 1

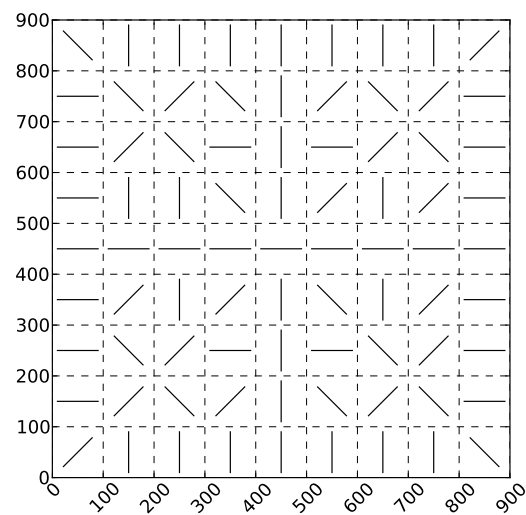

(d) Ply 4

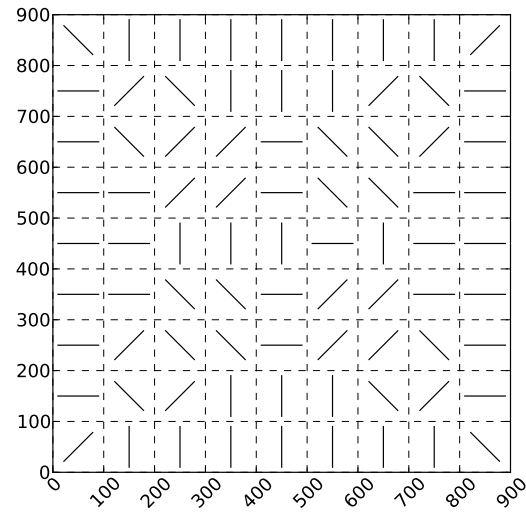

(b) Ply 2

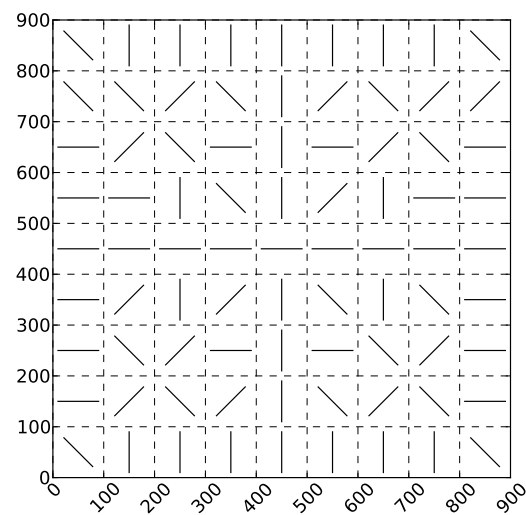

(e) Ply 5

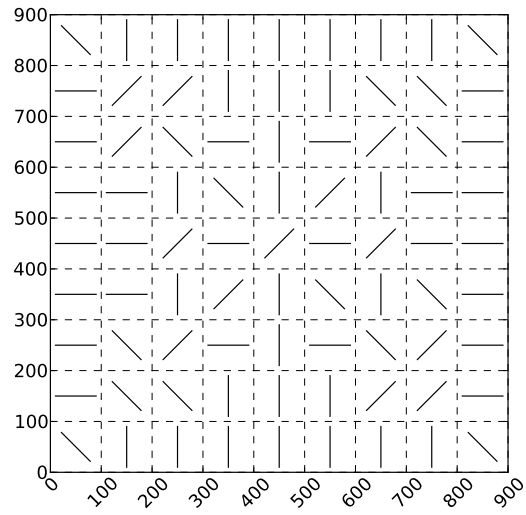

(c) Ply 3

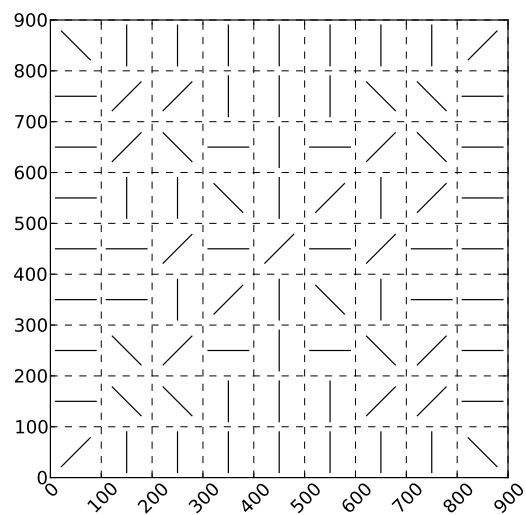

(f) Ply 6

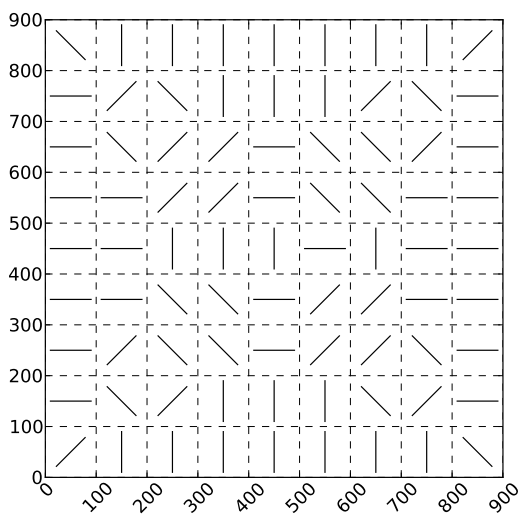

(g) Ply 7

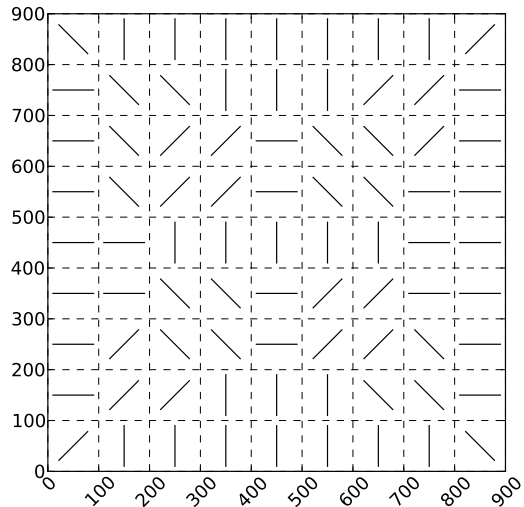

(h) Ply 8

Figure 4: The compliance minimization results for an 8-ply laminate. 


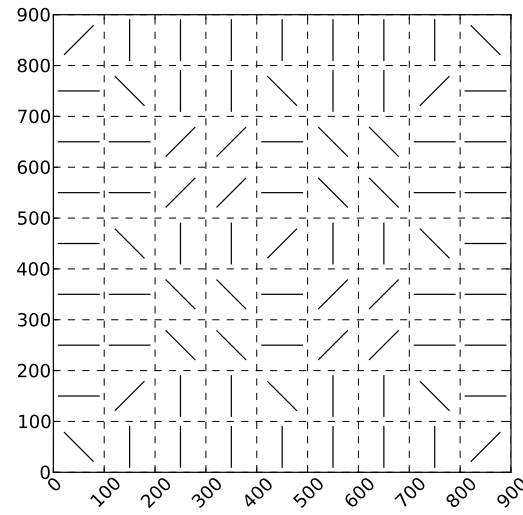

(a) Ply 1

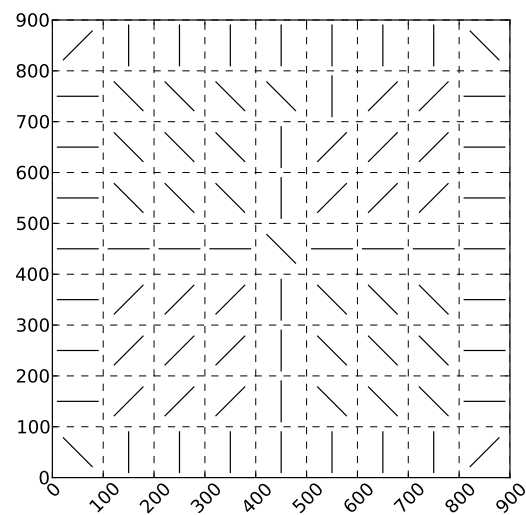

(d) Ply 4

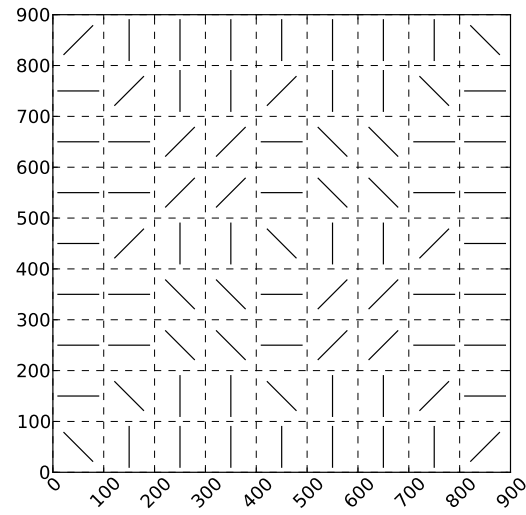

(b) Ply 2

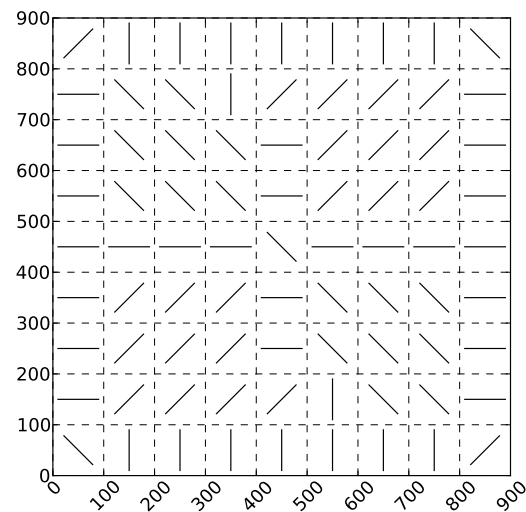

(e) Ply 5

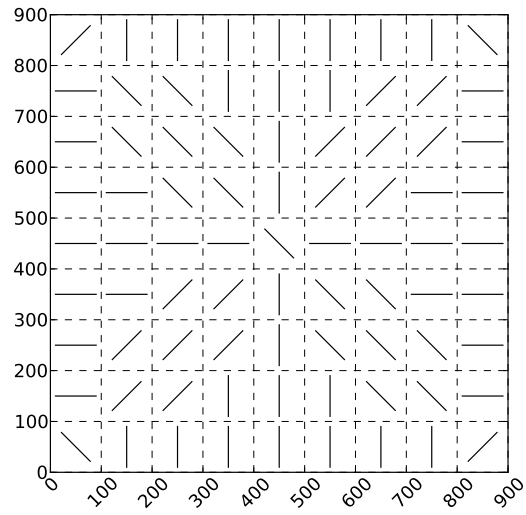

(c) Ply 3

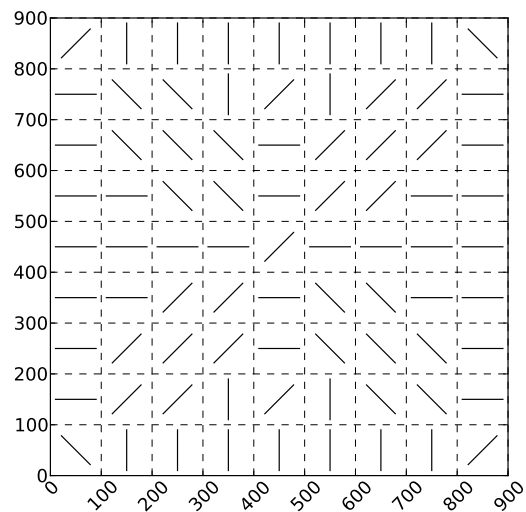

(f) Ply 6

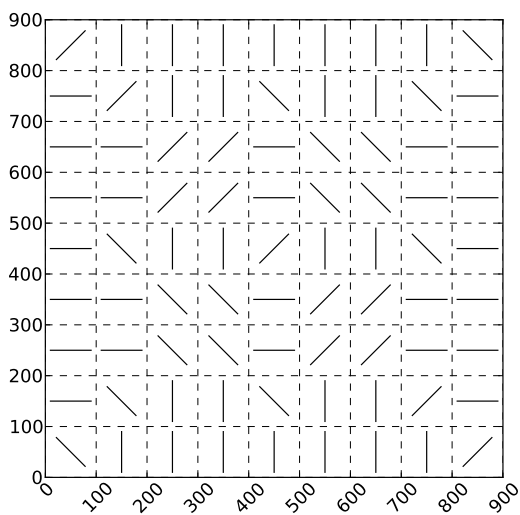

(g) Ply 7

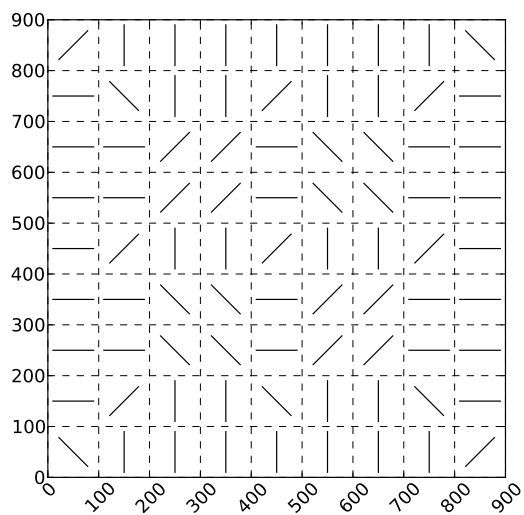

(h) Ply 8

Figure 5: The compliance minimization results for an 8-ply laminate with the adjacency constraints. 
problem, which we denote FailOpt $(P, \gamma, \epsilon, \tau)$, can be written as follows:

$$
\begin{aligned}
\operatorname{maximize} & \lambda-\gamma \mathbf{e}^{T}\left(\mathbf{e}-\mathbf{c}_{s}(\mathbf{x})\right) \\
\text { w.r.t. } & \lambda, \mathbf{x} \geq 0 \\
\text { s.t. } & \mathbf{K} \mathbf{u}_{m}=\lambda \mathbf{f}_{m} \quad m=1,2 \\
& \mathrm{KS}_{i j}\left(F^{(i j)}(\boldsymbol{\sigma})\right) \leq 1 \quad \forall m, i, j \\
& \mathbf{d}(x) \leq \tau \\
& \mathbf{A}_{w} \mathbf{x} \equiv \mathbf{e}
\end{aligned}
$$

where $P$ is the SIMP parameter, $\gamma$ is the penalty parameter, $\epsilon$ is the failure-penalization, and $\tau$ controls the infeasibility of the complementarity constraints. The goal is to maximize the load factor $\lambda$ subject to the failure criterion, and, when applicable, the adjacency constraints $\mathbf{d}(\mathbf{x})$.

We solve the problem FailOpt $(P, \gamma, \epsilon, \tau)$ for non-decreasing, $P, \gamma$ and $\epsilon$, and non-increasing $\tau$. The precise sequence of parameters chosen in the continuation approach presented here are listed in Table 3 . Note that the classical methods of forcing a 0-1 solution by increasing $P$ and $\epsilon$ do not produce a $0-1$ solution in this case, for this set of parameters. Only after the penalty parameter $\gamma$ is further increased and the parameter $\tau$ is decreased do the solutions converge to $0-1$ points. Furthermore, increasing the additional computational cost, as measured by function and gradient evaluations, is moderate for the optimization problem with no additional parameters. This suggests that increasing the parameter $\gamma$ has not resulted in poor conditioning of the optimization problem.

Figure 6 shows the ply angles of the optimization without adjacent ply-angle constraints, and Figure 7 shows the results of the optimization. Note that in both cases the laminates are not symmetric.

\begin{tabular}{lcccccccc}
\hline Iteration & $P$ & $\gamma$ & $\epsilon$ & $\tau$ & $\lambda$ & Infeasibility & $\begin{array}{l}\text { Function } \\
\text { evaluations }\end{array}$ & $\begin{array}{l}\text { Gradient } \\
\text { evaluations }\end{array}$ \\
\hline \multicolumn{7}{l}{ No adjacency constraints } \\
\hline 1 & 1 & 0 & 0.3 & - & 2.13885899 & 20.384 & 200 & 55 \\
2 & 2 & 0 & 0.3 & - & 2.03593877 & 2.8265 & 99 & 27 \\
3 & 2 & $10^{-4}$ & 0.3 & - & 2.03593789 & 2.8094 & 8 & 4 \\
4 & 2 & $10^{-3}$ & 0.3 & - & 2.03584405 & 2.3958 & 39 & 11 \\
5 & 2 & $10^{-2}$ & 0.3 & - & 2.03422539 & 1.8176 & 74 & 18 \\
6 & 2 & $10^{-1}$ & 0.3 & - & 1.99492029 & 0.69431 & 34 & 5 \\
7 & 2 & 1 & 0.3 & - & 1.93001389 & $9.5479 \times 10^{-15}$ & 23 & 4 \\
\hline With adjacency constraints & & & & & 331 & 97 \\
\hline 1 & 1 & 0 & 0.3 & 0.5 & 2.12631323 & 39.516 & 200 & 75 \\
2 & 2 & 0 & 0.3 & 0.5 & 1.88779621 & 19.673 & 352 & 118 \\
3 & 2 & $10^{-4}$ & 0.3 & 0.25 & 1.87702320 & 13.476 & 344 & 114 \\
4 & 2 & $10^{-3}$ & 0.3 & 0.25 & 1.90108267 & 9.1455 & 125 & 30 \\
5 & 2 & $10^{-2}$ & 0.3 & 0.125 & 1.88274591 & 1.0598 & 28 & 7 \\
6 & 2 & $10^{-1}$ & 0.3 & 0.125 & 1.85466596 & 0.015639 & 15 & 3 \\
7 & 2 & 1 & 0.3 & 0.06125 & 1.85299178 & $1.6187 \times 10^{-13}$ & & \\
\hline
\end{tabular}

Table 3: Failure criteria minimization with and without adjacency constraints. Note that the infeasibility is calculated as $\left\|\mathbf{c}_{s}\left(\mathbf{x}_{n}^{*}\right)-\mathbf{e}\right\|_{1}$.

\section{Compliance minimization of an isotropic plate}

In this section, we present results of a compliance minimization study of a fully clamped isotropic plate subject to a uniform pressure load. In this problem we restrict the allowable set of thicknesses to $t=\{3,4,5,6,7\} \mathrm{mm}$. The plate under consideration is $320 \times 320 \mathrm{~mm}$ and is fully clamped along all four edges and is loaded with a constant $100 \mathrm{kPa}$ pressure load. Instead of modeling the entire plate, we enforce symmetry in both the structural solution and in the design. We simulate only $1 / 4$ of the plate and impose symmetry boundary conditions. Additionally, we enforce 


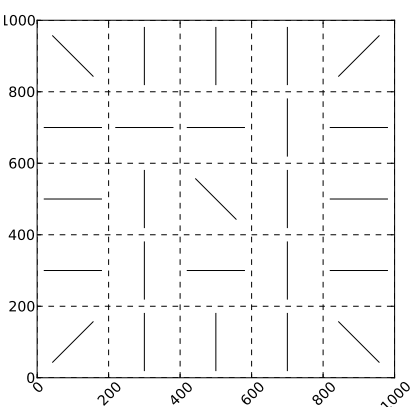

(a) Ply 1

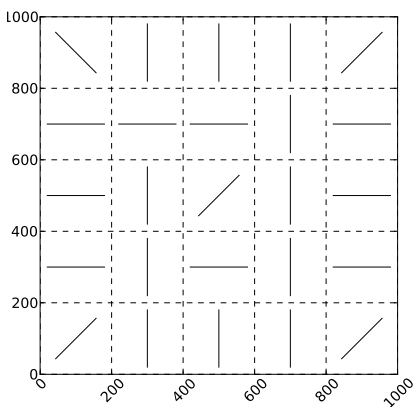

(d) Ply 4

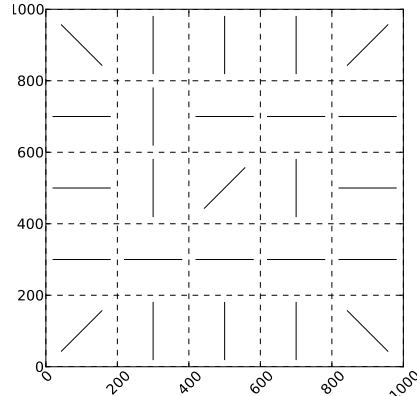

(b) Ply 2

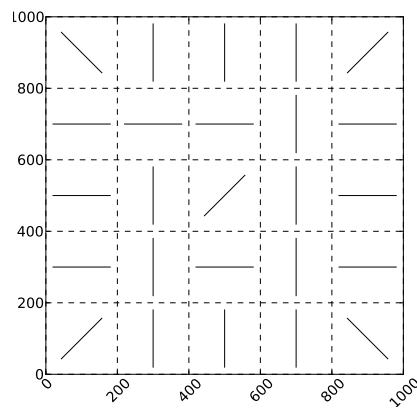

(e) Ply 5

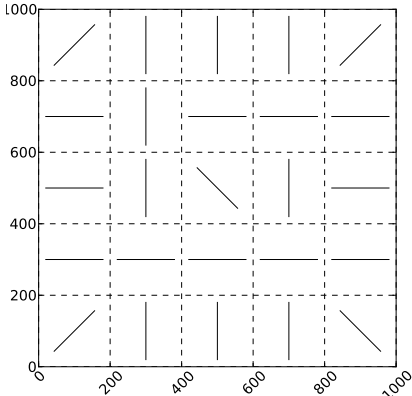

(c) Ply 3

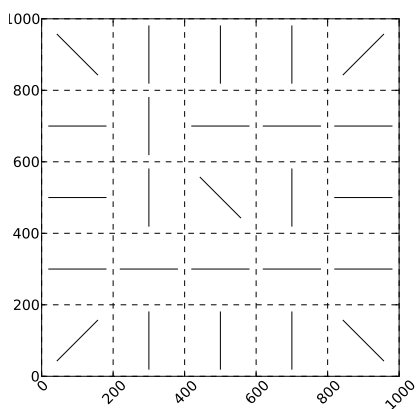

(f) Ply 6

Figure 6: The failure load maximization results for the 6-ply laminate.

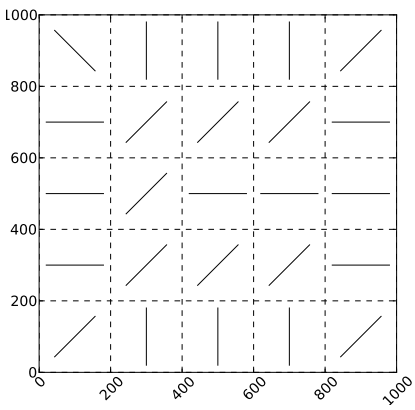

(a) Ply 1

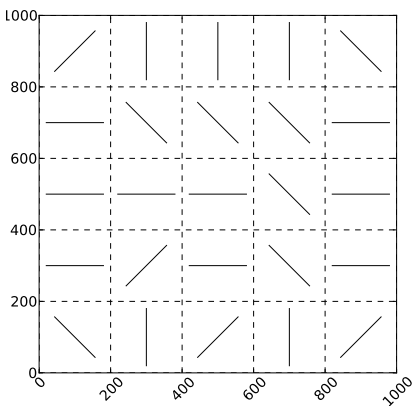

(d) Ply 4

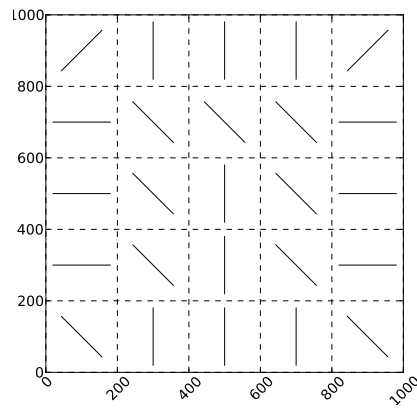

(b) Ply 2

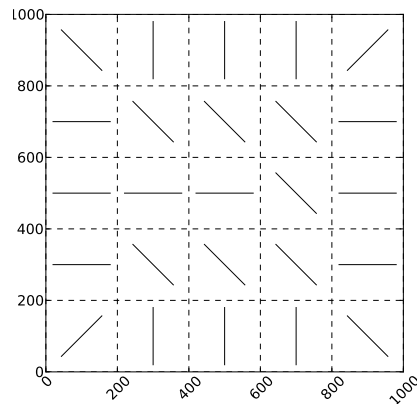

(e) Ply 5

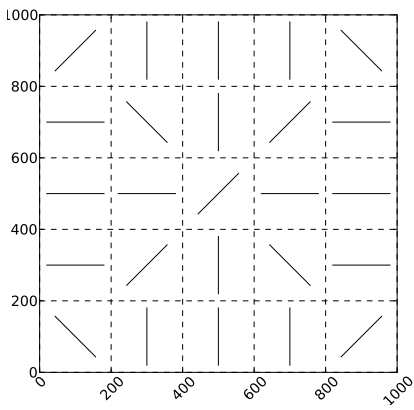

(c) Ply 3

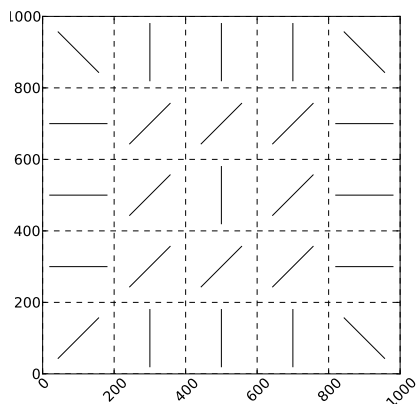

(f) Ply 6

Figure 7: The failure load maximization results for the 6-ply laminate with adjacency constraints. 
design symmetry across the diagonal of the plate. Finally, we enforce a mass constraint such that the plate has the same mass as a $5 \mathrm{~mm}$ constant-thickness plate with the same dimensions.

The $160 \times 160 \mathrm{~mm}$ quarter of the plate is modeled using $48 \times 48,3^{\text {rd }}$ order MITC9 shell elements. Each element is assigned an independent thickness. When using the discrete thickness formulation described above, this results in 1176 thickness variables and 1176 interpolation variables for a total of 2352 design variables. In addition, there are a total of 5880 thickness interpolation constraints associated with the discrete formulation, and one mass constraint.

The optimization formulation, which we denote $\operatorname{IsoCompOpt}(Q)$, is posed as follows:

$$
\begin{aligned}
\operatorname{minimize} & \frac{1}{2} \mathbf{u}^{T} \mathbf{K u} \\
\text { w.r.t. } & \mathbf{t} \\
\text { s.t. } & \mathbf{K u}=\mathbf{f} \\
& m(\mathbf{t})=5 \\
& \mathbf{g}(\mathbf{t}) \leq 0
\end{aligned}
$$

here $m(\mathbf{t})$ is the mass per unit area of the plate, $\mathbf{K}$ and $\mathbf{f}$ are the stiffness and consistent force vector, and $\mathbf{g}(\mathbf{t})$ are the discrete thickness interpolation constraints (21).

We solve the problem IsoCompOpt $\left(Q_{n}\right)$ for a series of $Q_{n}$, starting each new optimization from the solution obtained at the previous step. The sequence $Q_{n}$ and the optimization results are summarized in Table 4 . In this table, the infeasibility is measured using the following formula:

$$
\text { Infeasibility }=\sum_{k} \min _{i}\left(\left|t_{k}^{\prime}-t_{i}\right|\right),
$$

which measures the distance to the nearest discrete solution. Table 4 also shows the mass and compliance obtained by rounding the solution to the nearest discrete point. At this rounded point the mass constraint may be violated and the compliance may be lower if additional mass is added.

\begin{tabular}{|c|c|c|c|c|c|c|c|}
\hline Iteration & $Q$ & Compliance & Infeasibility & $\begin{array}{l}\text { Rounded } \\
\text { compliance }\end{array}$ & $\begin{array}{l}\text { Rounded } \\
\text { mass }\end{array}$ & $\begin{array}{l}\text { Function } \\
\text { evaluations }\end{array}$ & $\begin{array}{l}\text { Gradient } \\
\text { evaluations }\end{array}$ \\
\hline \multicolumn{8}{|c|}{ Discrete thickness } \\
\hline 1 & 0 & 675.92 & 23.2 & 675.73 & 5.0017 & 117 & 32 \\
\hline 2 & 0.05 & 676.17 & 1.18 & 675.95 & 5.0009 & 74 & 23 \\
\hline 3 & 0.25 & 676.17 & $8.62 \times 10^{-14}$ & 676.17 & 5.0 & 32 & 13 \\
\hline \multicolumn{8}{|c|}{ Continuous thickness } \\
\hline 1 & - & 676.34 & 23.8 & 676.60 & 5.0 & 151 & 57 \\
\hline
\end{tabular}

Figure 8 shows the final solution for the discrete thickness optimization and an equivalent continuous optimization. Both the discrete and continuous solutions share similar characteristics. Both contain large portions of 3 and $7 \mathrm{~mm}$ thick areas. In both solutions, fingers extend between the boundaries of the 3 and $7 \mathrm{~mm}$ thick regions. In Table 4 , the compliance values are very close, and in fact, the discrete solution is slightly lower than the continuous solution. This indicates that both solutions are, in all likelihood, local minima.

Table 4: Discrete thickness selection for compliance minimization for both the discrete formulation and an equivalent continuous optimization. The columns "Rounded compliance" and "Rounded mass" refer to the compliance and mass of the solution rounded to the nearest discrete point.

\section{Maximum von Mises stress minimization of an isotropic plate}

In this section, we present results for a maximum normalized von Mises stress minimization study for a fully clamped isotropic plate subject to a pressure load. Here, the objective is to minimize the maximum normalized von Mises stress in a square plate subject to a mass constraint. The square plate is $320 \times 320 \mathrm{~mm}$ and subject to a constant pressure load of $100 \mathrm{kPa}$. The plate is fully clamped along the edges, however, we apply symmetry conditions and model only $1 / 4$ of the plate. Furthermore, we enforce design symmetry across the diagonal of the plate. The mass is constrained to be the same as that of $5 \mathrm{~mm}$ thick plate of constant-thickness with the same dimensions. 


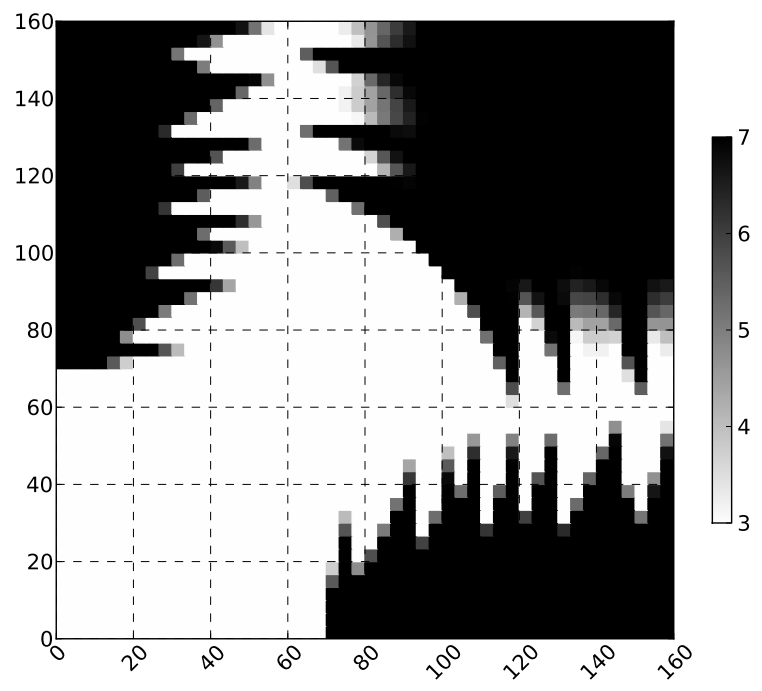

(a) Continuous

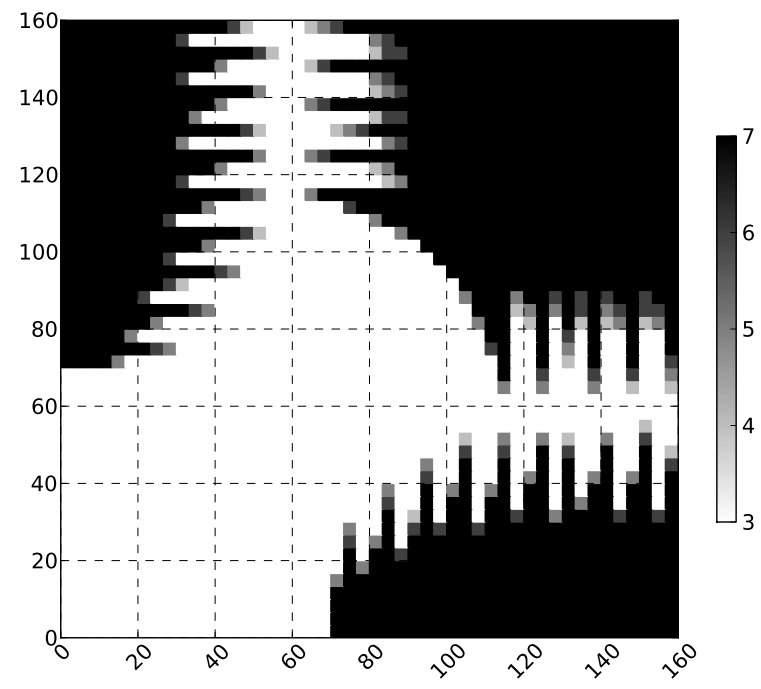

(b) Discrete

Figure 8: A comparison between the continuous and discrete compliance minimization results.

We split the plate into $16 \times 16$ design segments, each of which have a constant thickness. These segments are each modeled using $3 \times 3,3^{\text {rd }}$ order MITC9 elements, resulting in a finite-element mesh with 2304 finite-elements, 9409 nodes and over 56000 degrees of freedom. Due to the enforced diagonal symmetry, there are 136 design segments and 272 design variables.

For this optimization problem, we restrict the allowable thickness to the discrete set of values $\mathcal{T}=\{3,4,5,6,7\}$ $\mathrm{mm}$. We compare the discrete optimization results to a continuous solution obtained by minimizing the maximum von Mises stress with thickness $3 \leq t \leq 7 \mathrm{~mm}$.

As in the previous examples, we use a continuation method. We use the following optimization problem formulation, which we denote $\operatorname{IsoFailOpt}(Q)$ :

$$
\begin{aligned}
\operatorname{minimize} & s \\
\text { w.r.t. } & s, \mathbf{t} \\
\text { s.t. } & \mathbf{K u}=\mathbf{f} \\
& \mathrm{KS}_{i}\left(\sigma_{v M} / \sigma_{y s}\right) \leq s \quad \forall i \\
& \mathbf{g}(\mathbf{t}) \leq 0
\end{aligned}
$$

In this problem, $s$ is an additional bound variable [31] that represents the upper bound on the maximum value of the KS function over all design segments.

Table 5 lists the sequence of optimization results and parameters used in the continuation method. Note that the infeasibility measure is the same formula used in Equation (26). The infeasibility of the solution as measured by Equation (26) does not fully converge to zero. This is due to the inexact nature of the penalty function. In this problem it is advantageous to have some partially converged segments that are below the maximum von Mises stress. These partially converged segments reduce the maximum elsewhere, but incur a strength-to-weight penalty.

Figure 9 shows a comparison between the discrete and continuous results. In the continuous solution, the thickness varies gradually from the center of the plate towards the edges, whereas the abrupt changes in thickness are clearly visible in the discrete solution. Both the continuous and the discrete solutions share similar characteristics.

\section{E. Buckling optimization of a stiffened panel}

In this section, we present results of a series of optimizations performed for a stiffened panel. The geometry of the stiffened panel is shown in Figure 10. The panel consists of four equally spaced stiffeners aligned along the $x$-direction. 


\begin{tabular}{|c|c|c|c|c|c|c|c|}
\hline Iteration & $Q$ & $s$ & Infeasibility & $\begin{array}{l}\text { Rounded } \\
\max _{i} \mathrm{KS}_{i}\left(\sigma_{v M} / \sigma_{y s}\right)\end{array}$ & $\begin{array}{l}\text { Rounded } \\
\text { mass }\end{array}$ & $\begin{array}{l}\text { Function } \\
\text { evaluations }\end{array}$ & $\begin{array}{l}\text { Gradient } \\
\text { evaluations }\end{array}$ \\
\hline \multicolumn{8}{|c|}{ Discrete thickness } \\
\hline 1 & 0 & 0.259370 & 14.5 & 0.269394 & 5.0195 & 343 & 39 \\
\hline 2 & 0.05 & 0.260038 & 8.53 & 0.270023 & 5.0039 & 151 & 48 \\
\hline 3 & 0.25 & 0.261302 & 2.47 & 0.266075 & 4.9922 & 166 & 48 \\
\hline \multicolumn{8}{|c|}{ Continuous thickness } \\
\hline 1 & - & 0.259565 & 30.3 & 0.266384 & 4.9922 & 203 & 32 \\
\hline
\end{tabular}

Table 5: Comparison between the a normalized von Mises stress minimization for both continuous and discrete thickness optimization approaches. Note that the columns "Rounded $\max _{i} \mathrm{KS}_{i}\left(\sigma_{v M} / \sigma_{y s}\right)$ " and "Rounded mass" refer to the value of the maximum stress constraint and the mass at the nearest discrete point from the solution.

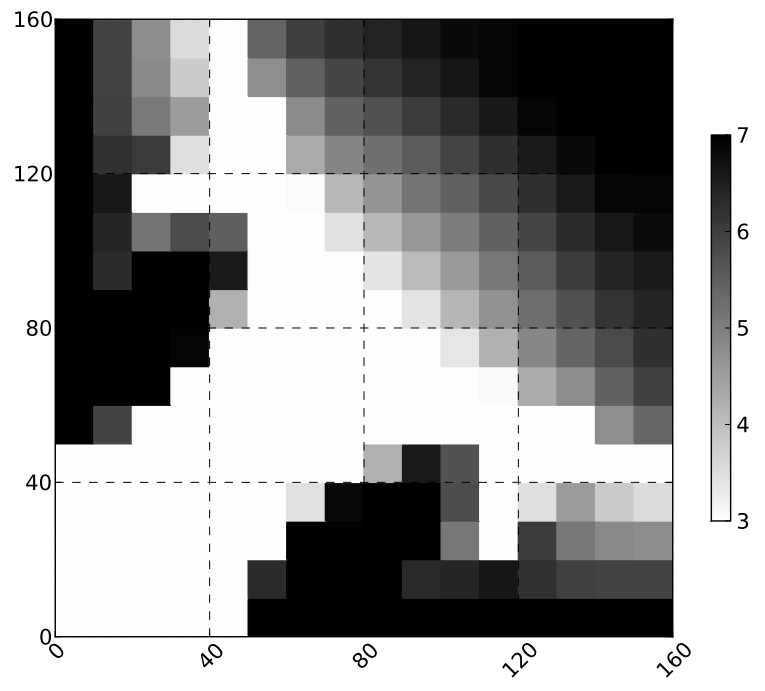

(a) Continuous

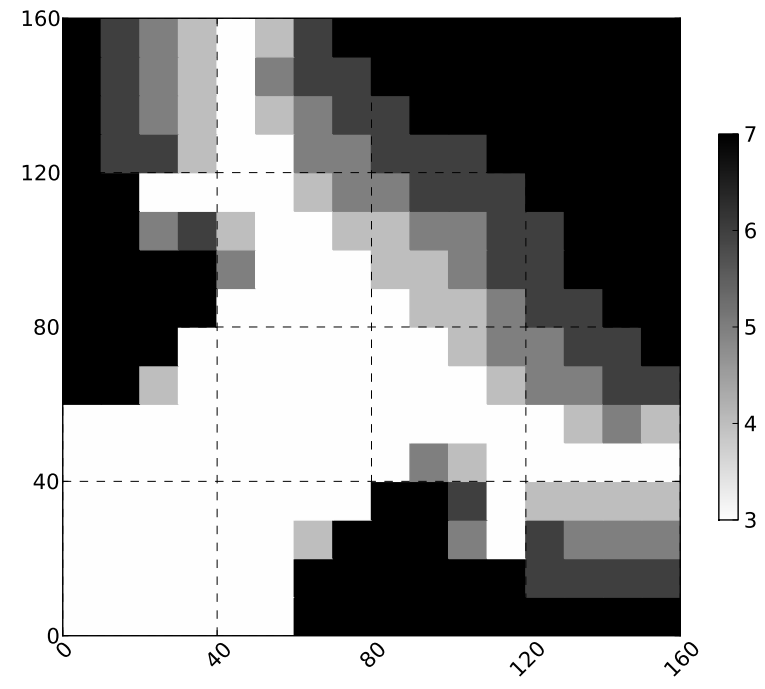

(b) Discrete

Figure 9: A comparison between the continuous von Mises stress minimization results and the DTO results for the same problem. 
The panel is subjected to a prescribed end shortening in the $x$-direction such that $u=-\Delta$, at $x=L_{x}$, and $u=0$ at $x=0$. The displacements along the $y=0$ and $y=L_{y}$ edges of the skin are simply supported, while the stiffeners are permitted to elongate in the $z$-direction at the ends $x=0$ and $x=L_{x}$.

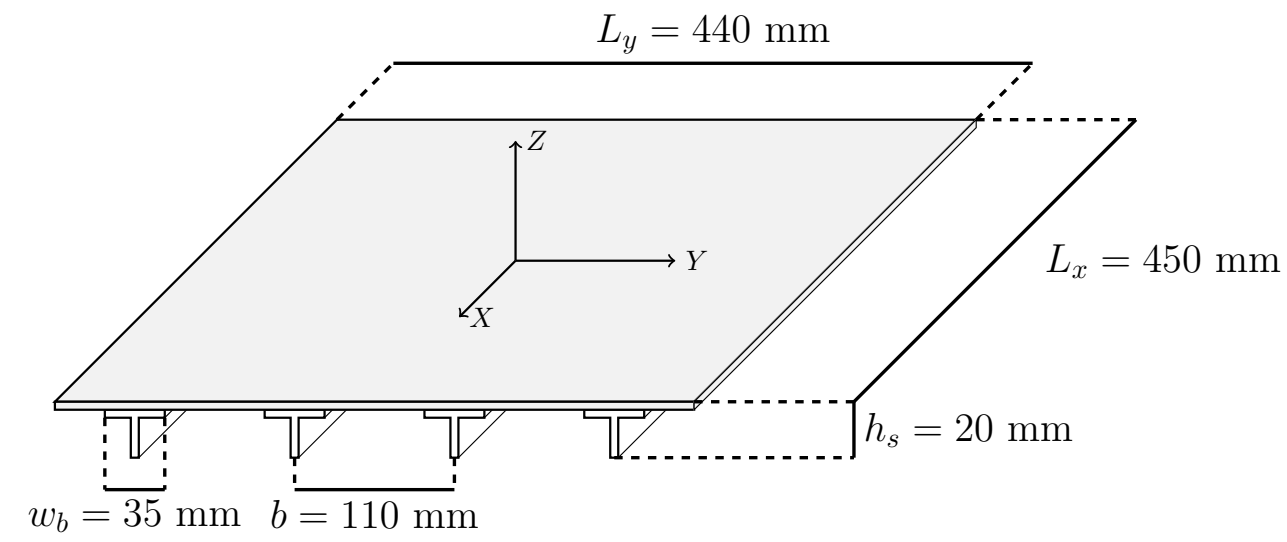

Figure 10: The dimensions of the buckling optimization problem formulation.

We model the stiffened panel using a finite-element mesh consisting of $15840,3^{\text {rd }}$ order MITC 9 shell elements: 120 along the length, 128 in the transverse direction and 5 through the depth of each stiffener. The finite-element model contains just over 383000 degrees of freedom. We solve the linear buckling eigenvalue problem on 16 processors using the parallel capabilities in TACS [29]. The buckling calculation involves a determination of the initial solution path $\mathbf{u}_{p}$, due to forces caused by the prescribed end-shortening $\mathbf{f}_{p}$ :

$$
\mathbf{K} \mathbf{u}_{p}=\mathbf{f}_{p}
$$

Once the load path $\mathbf{u}_{p}$ is calculated, the following linearized buckling eigenvalue analysis is used to determine the critical buckling load:

$$
\mathbf{K u}+\Delta_{c r} \mathbf{G}\left(\mathbf{u}_{p}\right) \mathbf{u}=0,
$$

where $\mathbf{G}\left(\mathbf{u}_{p}\right)$ is the geometric stiffness matrix which is a function of the initial load path.

The sensitivities of the eigenvalues $d \Delta_{c r} / d \mathbf{x}$ can be determined if the derivatives of the stiffness matrix and geometric stiffness matrices are known [32]. The main computational cost is in computing the sensitivities of geometric stiffness matrix which requires a contribution from the load-path computation. The derivative of the geometric stiffness matrix can be found as follows:

$$
\begin{aligned}
\frac{d \mathbf{G}}{d \mathbf{x}} & =\frac{\partial \mathbf{G}}{\partial \mathbf{x}}+\frac{\partial \mathbf{G}}{\partial \mathbf{u}_{p}} \cdot \frac{d \mathbf{u}_{p}}{d \mathbf{x}}, \\
& =\frac{\partial \mathbf{G}}{\partial \mathbf{x}}+\frac{\partial \mathbf{G}}{\partial \mathbf{u}_{p}} \cdot \mathbf{K}^{-1} \frac{\partial}{\partial \mathbf{x}}\left[\mathbf{f}_{p}-\mathbf{K} \mathbf{u}_{p}\right],
\end{aligned}
$$

where the operator $(\cdot)$ is used to denote a tensor-vector inner product.

In this buckling problem, we assume that the geometry of the panel and the number of plies at $0^{\circ}, \pm 45^{\circ}$, and $90^{\circ}$ are fixed. The objective is to maximize the critical end-shortening subject to various constraints on the laminate stacking sequence. Here we fix the thicknesses of the skin, stiffener-base and stiffener at 24,30 and 20 plies respectively. The number of plies in the skin and the stiffener at $0^{\circ}, 45^{\circ},-45^{\circ}$ and $90^{\circ}$ are $8,6,6$ and 4 , and 10, 4, 4 and 2 respectively. The outer six plies on both sides of the stiffener form the bottom 6 plies of the stiffener pad. An additional 8 plies are added in the middle of the stiffener. Note that the laminates in the skin and stiffener are balanced, while the laminate of the stiffener-base may be non-symmetric.

We impose the following linear constraint for the skin and stiffener layup to prescribe the number of plies at a given angle $\theta_{i}$ :

$$
\sum_{j=1}^{N} x_{i j k}=p_{i k}, \quad i=1, \ldots, 3, \quad k=1, \ldots, 4,
$$

where $p_{i k}$ are the number of plies in component $i$ at ply angle $\theta_{k}$. Matrix-cracking can occur in laminates when several contiguous plies are at the same angle [18]. To obtain laminate sequences that do not contain more than four repeated 
plies, we enforce the following linear inequality constraint:

$$
\sum_{j=p}^{p+5} x_{i j k} \leq 4, \quad i=1, \ldots, 4 \quad p=1, \ldots, N-5 .
$$

This constraint ensures that no more than four of the same plies are active over a five ply range.

\begin{tabular}{lrrrr}
\hline & Case A & Case B & Case C & Case D \\
\hline Design variables & & & & \\
\hline Skin ply identity & 96 & 48 & 96 & 48 \\
Stiffener ply identity & 40 & 40 & 40 & 40 \\
\hline Total & 136 & 88 & 136 & 88 \\
\hline Constraints & & & & \\
\hline Linear DMO $\left(\mathbf{A}_{w} \mathbf{X} \equiv \mathbf{e}\right)$ & 34 & 22 & 34 & 22 \\
Ply fraction $(\mathbf{B x}=\mathbf{p})$ & 8 & 8 & 8 & 8 \\
Contiguity constraint $(\mathbf{C x} \leq 4 \mathbf{e})$ & - & - & 120 & 88 \\
\hline Total & 42 & 30 & 162 & 118 \\
\hline
\end{tabular}

Table 6: Design problem summary for the buckling optimization studies

We examine four different lamination stacking sequence problems:

Case A Non-symmetric skin, symmetric stiffener

Case B Symmetric skin and stiffener

Case C Non-symmetric skin, symmetric stiffener and no more than four contiguous plies at the same angle

Case D Symmetric skin and stiffener and no more than four contiguous plies at the same angle

Each of these optimization problems can be expressed in the following formulation which we denote BucklingOpt $(\gamma)$ :

$$
\begin{aligned}
\operatorname{maximize} & \Delta_{c r}-\gamma \mathbf{e}^{T}\left(\mathbf{e}-\mathbf{c}_{s}(\mathbf{x})\right) \\
\text { w.r.t. } & \mathbf{x} \geq \\
\text { s.t. } & \mathbf{K} \mathbf{u}_{p}=\mathbf{f}_{p} \\
& \mathbf{K u}+\Delta_{c r} \mathbf{G}\left(\mathbf{u}_{p}\right) \mathbf{u}=0 \\
& \mathbf{B x}=\mathbf{p} \\
& \mathbf{C x} \leq 4 \mathbf{e} \\
& \mathbf{A}_{w} \mathbf{x} \equiv \mathbf{e}
\end{aligned}
$$

where $\mathbf{B x}=\mathbf{p}$ are the ply constraints (30) and $\mathbf{C x} \leq 4 \mathbf{e}$ are the contiguous ply constraints (31). Table 6 summarizes the design problems for the four buckling optimization cases. In each case, all the constraints are linear equality or linear inequality constraints.

For all cases, we fix $P=1$ and use a sequence of penalty parameters $\gamma_{1}=0, \gamma_{n}=10^{n-6}$ for $n \geq 2$. The optimization results for all cases are summarized in Table 7 and the layup designs are shown in Figure 11, for the skin, stiffener-base and stiffener laminates respectively. The non-symmetric laminate, Case A, converges to a slightly better design than the symmetric laminate, Case B. Both cases share the same stiffener layup, with a slightly different skin layup. For the skin layup of both Case A and Case B, the $0^{\circ}$ plies are placed in the middle, $90^{\circ}$ plies on the exterior and $\pm 45^{\circ}$ placed in between. The difference between Cases $\mathrm{A}$ and $\mathrm{B}$ is that for Case $\mathrm{A}$, an additional $90^{\circ}$ ply is placed at the bottom of the skin, the $0^{\circ}$ plies are offset from the middle and the arrangement of the $\pm 45^{\circ}$ plies is altered. This arrangement of ply angles is used to suppress the overall buckling mode which involves both the skin and stiffeners. Figure 13 shows the buckling mode for Case A. All designs exhibit the same overall buckling mode.

Neither Case C nor Case D converge to a 0-1 solution for the given sequence of $\gamma_{n}$. From Table 7, it is clear that both solutions converge to a local minima, since for a sufficiently large value of the penalty parameter, $\gamma$, both 


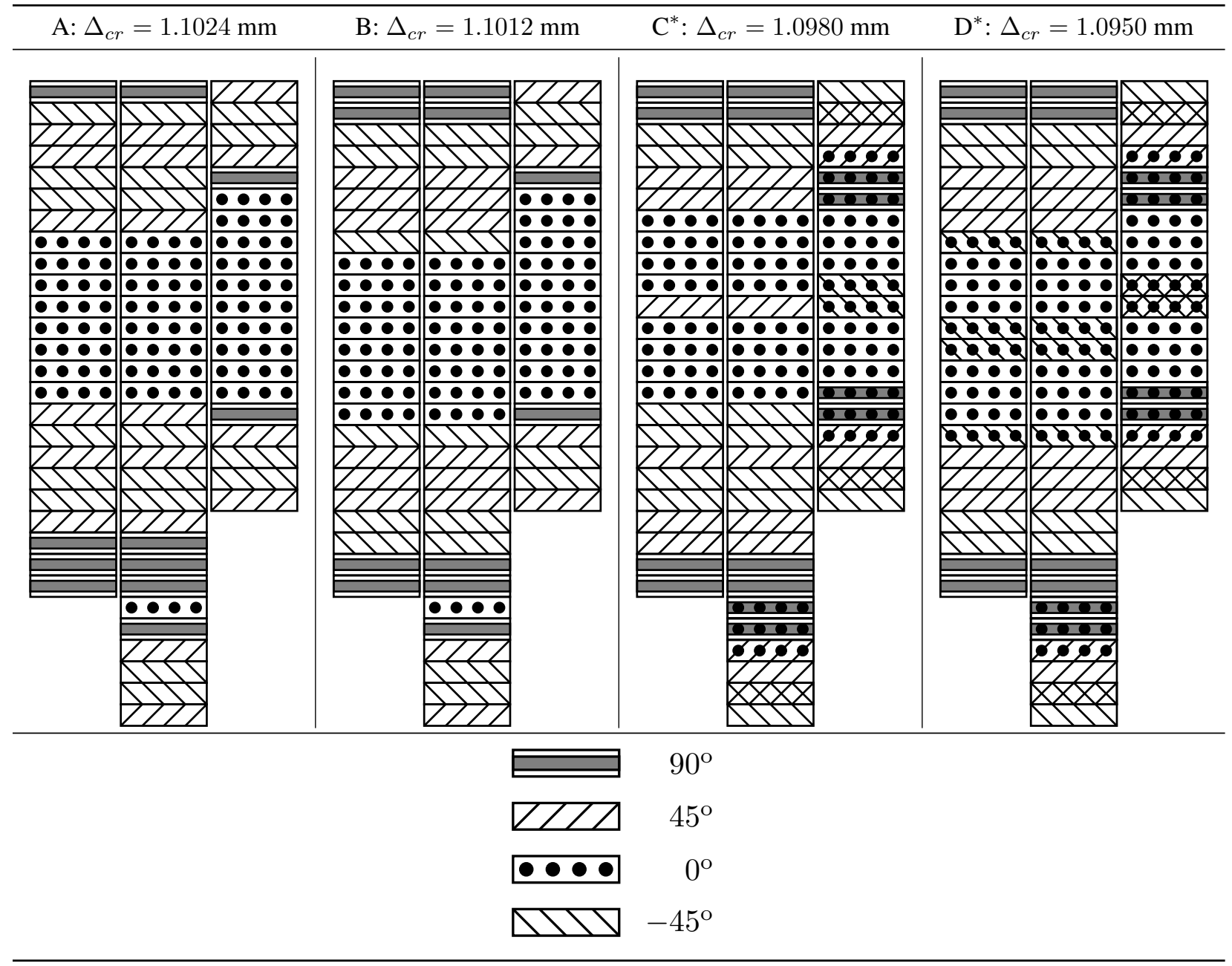

Figure 11: The optimal ply angle sequences for the buckling optimization problems. Each solution shows the skin, stiffener-base and skin layups respectively. Note that Case C and Case D have converged to local minima in which multiple plies are active. 


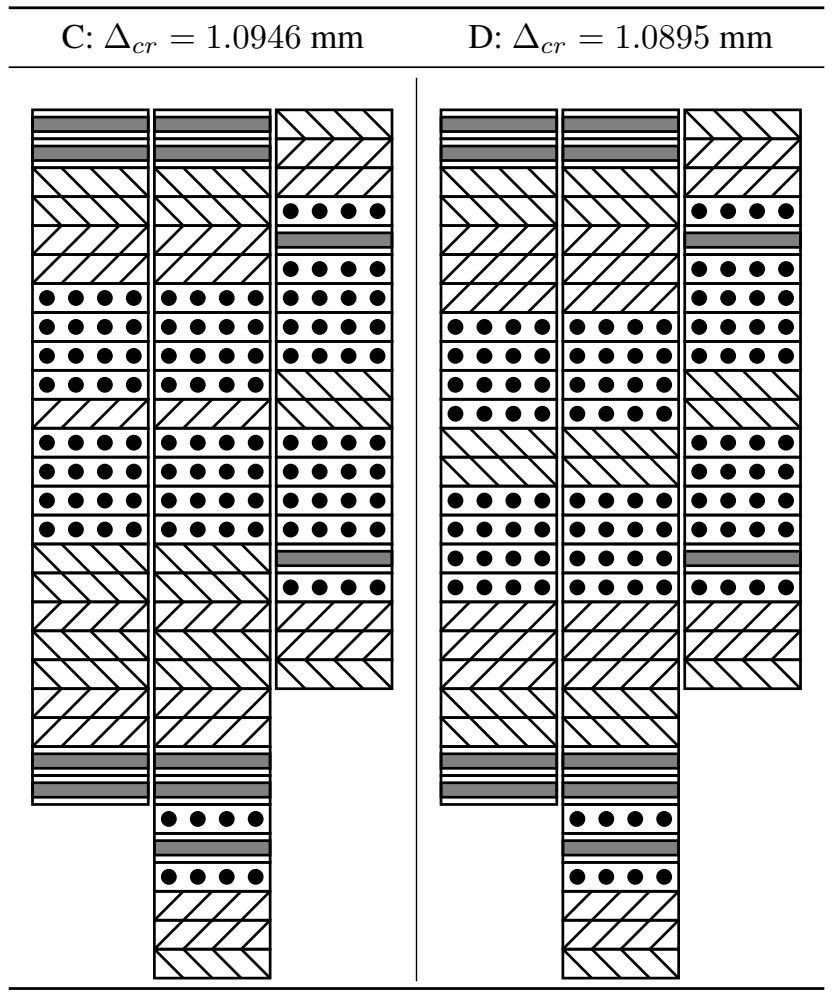

Figure 12: Ply angle sequences for feasible 0-1 points near the local minima for Case C and Case D.

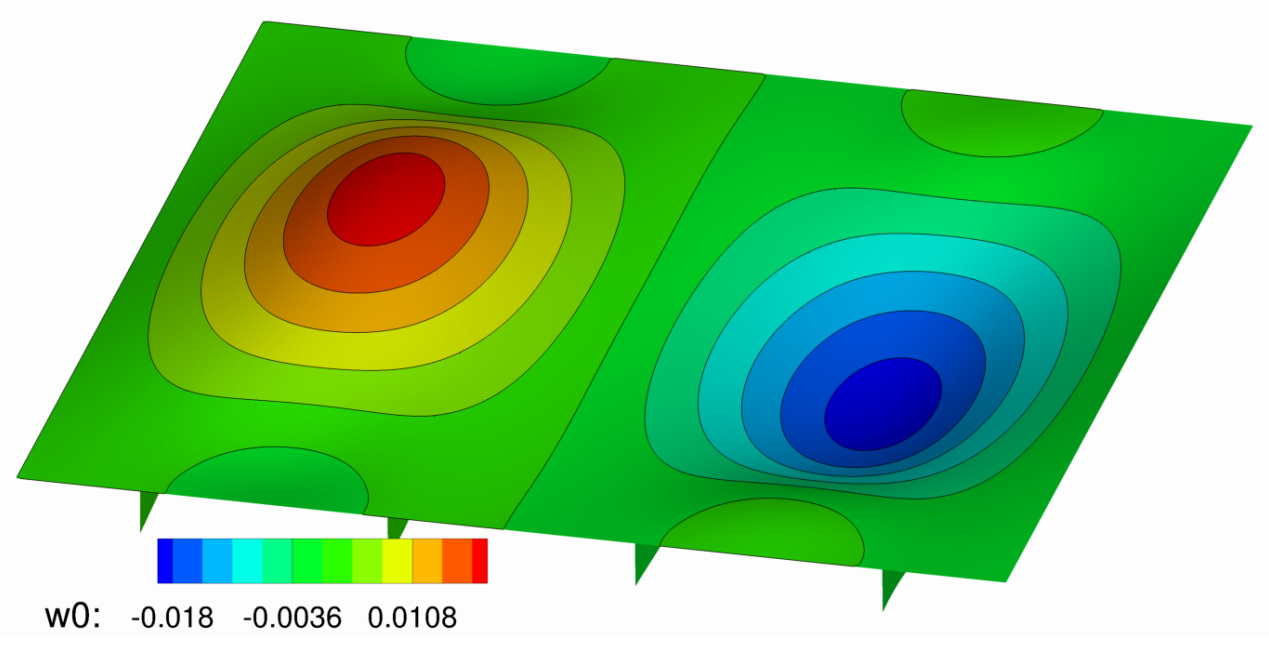

Figure 13: The lowest buckling eigenmode for Case A. Note that the other cases exhibit similar eigenmodes. 
objectives are negative. Note that any feasible $0-1$ point would have a higher value of the objective since the penalty parameter term $\gamma\left\|\mathbf{c}_{s}\left(\mathbf{x}^{*}\right)-\mathbf{e}\right\|_{1}$ would be zero and the critical end-shortening $\Delta_{c r}$ is always positive. Figure 12 shows feasible designs for Case $\mathrm{C}$ and Case $\mathrm{D}$ that are close to the designs obtained using the laminate parametrization technique. Note that these designs have slightly lower values of the critical end-shortening $\Delta_{c r}$ than the designs shown in Figure 11.

\begin{tabular}{|c|c|c|c|c|c|c|c|}
\hline Iteration & $P$ & $\gamma$ & $\Delta_{c r}[\mathrm{~mm}]$ & Infeasibility & Objective & $\begin{array}{l}\text { Function } \\
\text { evaluations }\end{array}$ & $\begin{array}{l}\text { Gradient } \\
\text { evaluations }\end{array}$ \\
\hline \multicolumn{8}{|l|}{ Case A } \\
\hline 1 & 1 & 0 & 1.1050 & 8.6534 & 1.1050 & 65 & 18 \\
\hline 2 & 1 & $10^{-4}$ & 1.1050 & 1.5822 & 1.1048 & 296 & 73 \\
\hline 3 & 1 & $10^{-3}$ & 1.1050 & 1.5531 & 1.1034 & 18 & 7 \\
\hline 4 & 1 & $10^{-2}$ & 1.1024 & $2.8604 \times 10^{-11}$ & 1.1024 & 52 & 8 \\
\hline \multicolumn{8}{|l|}{ Case B } \\
\hline 1 & 1 & 0 & 1.1017 & 4.9689 & 1.1017 & 48 & 10 \\
\hline 2 & 1 & $10^{-4}$ & 1.1017 & 0.98425 & 1.1016 & 110 & 22 \\
\hline 3 & 1 & $10^{-3}$ & 1.1012 & $1.3323 \times 10^{-15}$ & 1.1012 & 24 & 6 \\
\hline \multicolumn{8}{|l|}{ Case $\mathrm{C}$} \\
\hline 1 & 1 & 0 & 1.0988 & 9.3145 & 1.0988 & 104 & 27 \\
\hline 2 & 1 & $10^{-4}$ & 1.0988 & 3.4822 & 1.0984 & 186 & 48 \\
\hline 3 & 1 & $10^{-3}$ & 1.0986 & 2.9704 & 1.0956 & 37 & 6 \\
\hline 4 & 1 & $10^{-2}$ & 1.0980 & 2.5 & 1.0730 & 6 & 2 \\
\hline 5 & 1 & $10^{-1}$ & 1.0980 & 2.5 & 0.84798 & 2 & 1 \\
\hline 6 & 1 & 1 & 1.0980 & 2.5 & -1.4020 & 2 & 1 \\
\hline \multicolumn{3}{|c|}{ 0-1 point close to $C$} & 1.0946 & & & & \\
\hline \multicolumn{8}{|l|}{ Case D } \\
\hline 1 & 1 & 0 & 1.0955 & 6.4458 & 1.0955 & 100 & 22 \\
\hline 2 & 1 & $10^{-4}$ & 1.0955 & 4.6348 & 1.0950 & 156 & 25 \\
\hline 3 & 1 & $10^{-3}$ & 1.0952 & 3.7187 & 1.0915 & 37 & 6 \\
\hline 4 & 1 & $10^{-2}$ & 1.0950 & 3.5572 & 1.0594 & 39 & 6 \\
\hline 5 & 1 & $10^{-1}$ & 1.0950 & 3.5572 & 0.73927 & 5 & 2 \\
\hline 6 & 1 & 1 & 1.0950 & 3.5572 & -2.4622 & 2 & 1 \\
\hline \multicolumn{3}{|c|}{ 0-1 point close to $\mathrm{D}$} & 1.0895 & & & & \\
\hline
\end{tabular}

Table 7: A summary of the continuation history for the buckling optimization cases. Note that the infeasibility is calculated as $\left\|\mathbf{c}_{s}\left(\mathbf{x}_{n}^{*}\right)-\mathbf{e}\right\|_{1}$ and the objective is calculated from $\Delta_{c r}^{*}-\gamma \mathbf{e}^{T}\left(\mathbf{e}-\mathbf{c}_{s}\left(\mathbf{x}_{\mathbf{n}}^{*}\right)\right)$.

We have tried various sequences of penalty parameters, $\gamma_{n}$, to try and avoid the local minima in Case $\mathrm{C}$ and Case D. In particular, we have tried more gradually increasing sequences of $\gamma_{n}$. However, we have found that these sequences all lead to the same local minima shown in Figure 11, if started from the point $\gamma_{1}=0$. Furthermore, we have found that it is essential to use $\gamma_{1} \neq 0$ to obtain a good final design, otherwise the design tends towards a poorer local minima.

The failure of Case $\mathrm{C}$ and Case $\mathrm{D}$ to converge to a $0-1$ point is due to the addition of the ply contiguity constraints (31). These constraints are designed to apply to the discrete 0-1 solution, but also apply to any intermediate continuous point. The contiguity constraints are linear and so are satisfied by SNOPT exactly at every design iteration. The addition of these constraints makes the intermediate design a local minima by linking the design variables in such a way that the objective increases along all paths to a 0-1 point. For sufficiently large values of $\gamma$, the penalty term overwhelms the end-shortening, and the design problem is to essentially minimize $\left\|\mathbf{c}_{s}(\mathbf{x})-\mathbf{e}\right\|_{1}$, subject to the linear constraints. We found that even for $\gamma>10^{3}$, that the combination of linear inequality constraints were sufficient to keep the solution at the given local minima.

This example demonstrates that the proposed penalization approach ensures that the $0-1$ points are local minima, 
for sufficiently large values of $\gamma$. However, the proposed technique does not guarantee that other local minima, located away from 0-1 points, are eliminated from the design space.

\section{Wing-box optimization}

In this section, we present an application of the laminate parametrization presented in Section III, to the optimization of a wing-box, subject to aerodynamic loads. We consider both local buckling and failure constraints using a global-local analysis. In this analysis, the global model is a finite-element model with smeared stiffeners, while the local panel models consist of finite-strip models of the flat, stiffened-panels with discrete stiffeners. The finite-strip models are used to calculate an allowable buckling-free envelope and are also used to compute the equivalent smeared stiffness of the panels for the global finite-element model. The global finite-element model is used to enforce failure constraints and determine the average loads on the local panels that are used within the buckling calculations.

Other authors have performed design studies for composite wing boxes using similar methods. Liu et al. [13] performed a two level, global-local optimization of a composite wing with unstiffened panels. In their approach, the global problem was used to size the panels and determine the number of layers at specified angles, while the local problem was used to maximize the local buckling loads. Later, Liu and Haftka [33] performed an equivalent single-level optimization using lamination parameters to validate the two-level design approach.

In this study, the sizing and layup sequence design of a wing-box are split into two separate steps. First, using continuous lamination parameters, we minimize the structural weight subject to material failure and local buckling constraints. Second, with fixed structural thicknesses, we maximize the failure and buckling margin, by varying the lamination stacking sequence with the proposed laminate parametrization.

The remainder of this section is structured as follows. In Section A, we describe the geometry of the wing-box structure and the design loads. In Section B, we present results from the lamination parameter-based mass minimization problem. Finally, in Section C, we present the results of the laminate stacking sequence optimization. This test case demonstrates that the proposed lamination parametrization technique can be used for non-trivial design cases.

\section{A. Geometry, loads and analysis}

The geometry of the wing in this study is roughly based on a 777-200. The wing has a $60.9 \mathrm{~m}$ span with a $13.6 \mathrm{~m}$ root chord and a tip chord of $2.09 \mathrm{~m}$. The Yehudi break is located at $30 \%$ of the semi-span. The wing structure consists of two spars, 44 chord-wise ribs and top and bottom skins stretching between the front and rear spars. The front spar and rear spars are located at 10\% and $70 \%$ chord offset from the leading edge. We only model the structural box and omit any leading and trailing edge structure. The geometry of the wing-box structure can be seen in Figure 15.

To simplify the study, we only use only two design loads to size the structure: a $2.5 \mathrm{~g}$ maneuver and a $-1 \mathrm{~g}$ maneuver load, both at full fuel loads. We take the mass of the aircraft to be $300000 \mathrm{~kg}$ at the maneuver conditions. We compute the aerodynamic loads using TriPan, a parallel three-dimensional panel code [29]. The loads are transferred to the global structural model using a consistent and conservative load transfer scheme based on the work of Brown [34].

The global finite-element model of the wing consists of $67584,3^{\text {rd }}$ order, MITC9 shell elements, with 266852 nodes and just over 1.6 million degrees of freedom. We place 30 elements chordwise along each panel and 14 spanwise. In addition, we place 12 elements through the depth of each rib and spar.

The finite-strip panel models consist of 4 repeating stiffener-bay segments. These are used to compute the overall buckling mode using a sin-series expansion in the axial direction. Further details can be found in references [35, 36]. The local stiffened panel models are used to predict both the critical axial load $N_{x, c r}$ and the critical shear load $N_{x y, c r}$. Following Stroud and Agranoff [37], these buckling loads are used to form an approximate buckling-free envelope as follows:

$$
\frac{N_{x}}{N_{x, c r}}+\frac{N_{x y}^{2}}{N_{x y, c r}^{2}} \leq 1
$$

Each of the local panel buckling analyses are computationally inexpensive, relative to the global finite-element analysis. However, many individual panel analyses are required to obtain the buckling envelopes for all panels within the structure. As a result, we distribute the panel analysis in parallel across all the structural processes. Instead of designing each stiffened panel in the wing-box independently, we link adjacent panels together in pairs of two. This design-linking reduces the number of design variables and the number of panel analyses required.

\section{B. Preliminary sizing using lamination parameters}

To obtain a preliminary thickness distribution, we minimize the mass of the wing-box, subject to the failure and local buckling constraints. In this optimization problem, we use lamination parameters to parametrize the laminate. This 
allows us to handle laminate thickness changes, while leaving the details of the laminate sequence design for the second optimization problem. In order to apply failure constraints within this design problem, we apply the Tsai-Wu failure criteria, at the top and bottom surfaces of the the laminate for a series of angles, $\Theta=\left\{-90^{\circ}+n 15^{\circ}\right\}$, for $n=1, \ldots, 12$. We collect these failure criteria into a single function using the KS aggregation function [30]. This approach provides an approximate failure envelope for the lamination parameters.

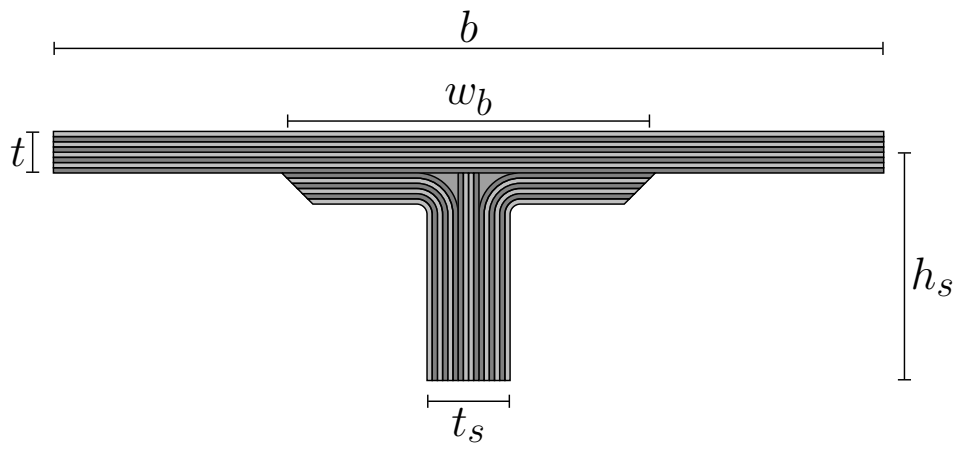

Figure 14: The panel geometry and thickness design variables used in the aerostructural optimization problem: stiffener pitch, $b$, stiffener-base or pad width, $w_{b}$, stiffener height, $h_{s}$, skin thickness, $t$, and stiffener thickness, $t_{s}$.

In the sizing problem, we fix the outer mold line of the wing and use only structure-specific geometric variables and structural thickness variables. In addition to the lamination parameters, we also include the stiffener pitch, stiffener pad-width, stiffener height, stiffener thickness and skin thickness as design variables. These thickness and geometric design variables are shown in Figure 14.

There are a total of 1938 design variables in the sizing problem. There are 23 design variables for each of the top and bottom skin panels: the stiffener height, the base width, and 6 lamination parameters and 1 thickness variable for each of the skin, stiffener-base and stiffener. This results in 1014 design variables for the top and bottom skins with 2 additional variables for the top stiffener pitch and bottom stiffener pitch which are linked across all panels. In addition, we use 6 lamination parameters and 1 thickness for each of the front and rear spar segments and each rib. This results in an additional 924 design variables.

There are a total of 970 nonlinear constraints in the sizing problem. There are 396 lamination parameter feasibility constraints for each skin, stiffener-base and stiffener, in each panel for the top and bottom skins. In addition, we use 396 lamination parameter feasibility constraints for the ribs, and front and rear spars. Finally, there are 44 buckling envelope constraints and 45 failure envelope constraints at the $2.5 \mathrm{~g}$ and $-1 \mathrm{~g}$ maneuver conditions respectively.

The sizing optimization problem can be written as follows:

$$
\begin{aligned}
\operatorname{minimize} & m(\mathbf{y}) \\
\text { w.r.t. } & \mathbf{y} \\
\text { s.t. } & \mathbf{K u}_{n}=\mathbf{f}_{n} \quad n=1,2 \\
& \mathrm{KS}_{i}(F(\boldsymbol{\sigma})) \leq 1 \quad \forall n, i \\
& \frac{N_{x}}{N_{x, c r}}+\frac{N_{x y}^{2}}{N_{x y, c r}^{2}} \leq 1 \quad \forall n, i \\
& \mathbf{h}(\mathbf{y}) \leq 1
\end{aligned}
$$

where $\mathbf{y}$ are the geometric, thickness, and lamination parameter design variables, $m(\mathbf{y})$ is the mass of the wing-box, $\mathbf{h}(\mathbf{y}) \leq 1$ are the lamination parameter feasibility constraints, and $n=1$ and $n=2$ correspond to the $2.5 \mathrm{~g}$ and $-1 \mathrm{~g}$ maneuver conditions respectively.

We solve Problem 34 on 48 processors in roughly 20 hours of wall time, with 1007 function and 287 gradient evaluations. Table 8 shows the mass breakdown of the solution. The top skins are significantly heavier than the bottom skins due, in part, to the buckling constraints and the lower strength of the composite under compression than under tension. Additional load cases, and consideration of the engine and landing-gear installation, would modify these results significantly.

Figure 15 shows the displaced structural solution for the mass-minimization problem, under the $2.5 \mathrm{~g}$ maneuver load. Figure 15 shows the force resultant out the span of the wing. Figure 16 shows the number of plies on the top 


\begin{tabular}{lrr}
\hline Component & Mass [kg] & Percentage \\
\hline Top skin & 10370 & 60.2 \\
Bottom skin & 4364 & 25.4 \\
Ribs & 718 & 4.2 \\
Spars & 1761 & 10.2 \\
\hline Total & 17213 & \\
\hline
\end{tabular}

Table 8: Mass component breakdown for the lamination-parameter-based sizing of the wing-box structure. These quantities are based on the total weight of the entire wing, not just the semi-span.

and bottom skins, and top and bottom stiffeners. Figure 17 shows the distribution of the stiffener heights for the top and bottom panels. Note that the top stiffener thicknesses are significantly higher than the thicknesses on the bottom, while the stiffener heights are larger on the bottom than on the top. Furthermore, an interesting interaction occurs near the Yehudi break where the top skin and stiffener thicknesses increase while the top stiffener height remains constant.

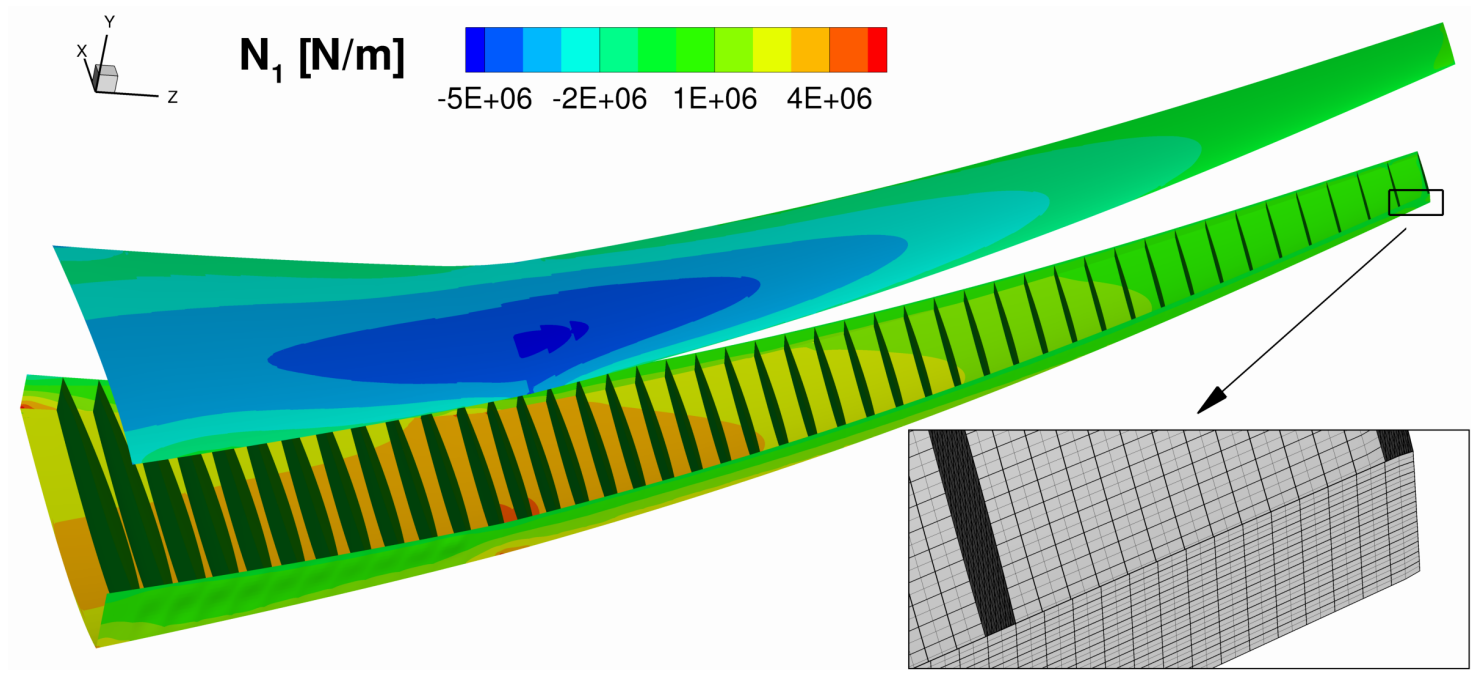

Figure 15: The displaced structural solution for the mass-minimization problem, under the $2.5 \mathrm{~g}$ maneuver load. The figure shows the force resultant out the span of the wing and the finite-element discretization with $3^{\text {rd }}$ order MITC9 shell elements.

\section{Layup optimization using the proposed lamination parametrization}

In this section, we present the results of the lamination stacking sequence design optimization using the proposed laminate parametrization method. In this optimization problem, we use the thickness distribution presented in Figure 16 and the stiffener height distribution presented in Figure 17. For the purposes of this optimization, these distributions are considered fixed.

In order to reduce the number of ply identity variables in the problem, we link the plies together into groups. For modeling purposes, the laminates must be symmetric and balanced. To enforce this condition, we select the following balanced ply groups: $\left[0_{4}^{\circ}\right],\left[ \pm 45_{2}^{\circ}\right]$ and $\left[90_{4}^{\circ}\right]$. We apply the laminate parametrization to these groups instead of the individual plies themselves and apply symmetry across the middle of the laminate. In this problem, we disregard issues of matrix cracking and do not apply a contiguity constraint. We do, however, require that a minimum of $\left\lfloor 0.15 N_{i}\right\rfloor$ plies of each ply angle must be present in each laminate, where $N_{i}$ are the number of plies in component $i$.

In this design, we use one lamination sequence for each of the following components: the upper skin, the upper skin stiffeners, the lower skin, and the lower skin stiffeners. Changes in thickness are accomplished by adding or removing plies from the outer-most portion of the laminate, symmetrically on both sides. This is designed to model a situation in which a layer in a single ply could be extended over the entire wing-box skin or stiffener. 


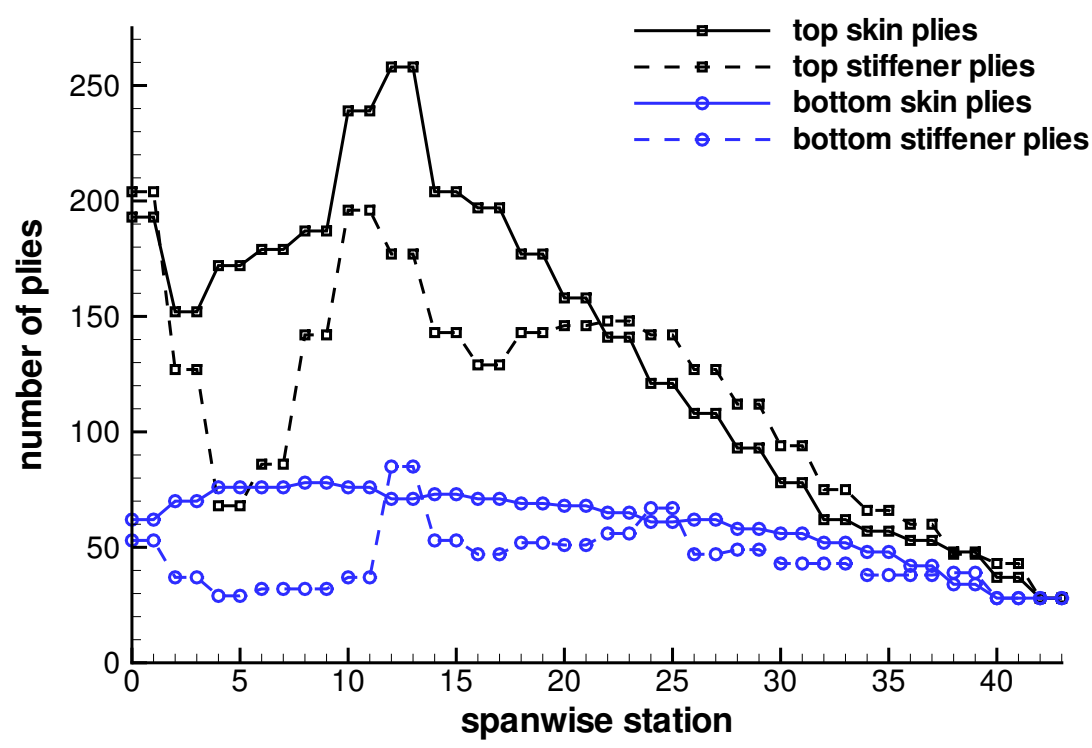

Figure 16: The number of plies on the top and bottom skin and stiffener.

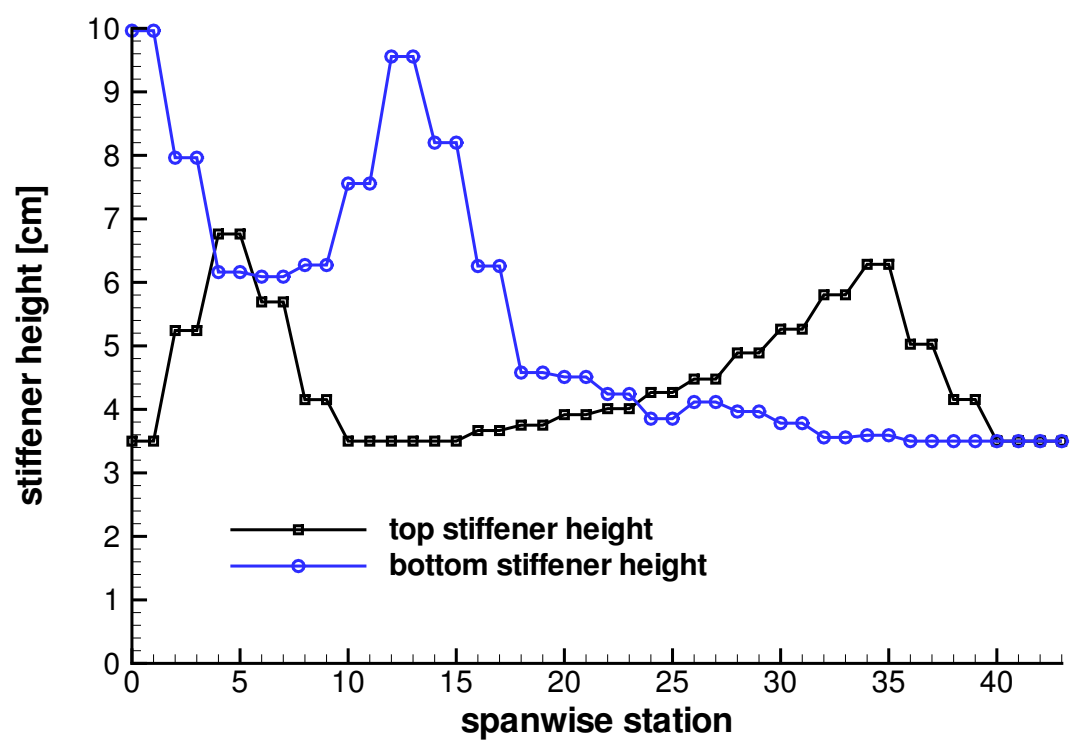

Figure 17: The number of plies on the top and bottom skin and stiffener. 
The objective of this optimization problem is to maximize the load factor $\lambda$, subject to failure and buckling constraints. We aggregate the failure constraints over each ply, in all the structural components, using the KS aggregation function. There are a total of 328 nonlinear constraints: 88 buckling constraints and $76 \mathrm{KS}$ failure constraints for each load case. There are a total of 229 variables: 3 ply selection variables for 76 plies, and 1 load factor variable.

The wing-box optimization problem can be expressed in the following form, which we denote WingBoxOpt $(\gamma)$ :

$$
\begin{aligned}
& \operatorname{maximize} \quad \lambda-\gamma \mathbf{e}^{T}\left(\mathbf{e}-\mathbf{c}_{s}(\mathbf{x})\right) \\
& \text { w.r.t. } \quad \lambda, \mathbf{x} \geq 0 \\
& \text { s.t. } \quad \mathbf{K} \mathbf{u}_{m}=\lambda \mathbf{f}_{m} \quad m=1,2 \\
& \mathrm{KS}_{j}\left(F^{(i j)}(\boldsymbol{\sigma})\right) \leq 1 \quad \forall m, j \\
& \frac{N_{x}}{N_{x, c r}}+\frac{N_{x y}^{2}}{N_{x y, c r}^{2}} \leq 1 \quad \forall n, i \\
& \text { Dx } \geq \mathbf{c} \\
& \mathbf{A}_{w} \mathbf{x} \equiv \mathbf{e}
\end{aligned}
$$

where $\mathbf{D x} \geq \mathbf{c}$ represents the constraint on the minimum ply content.

\begin{tabular}{lccccccc}
\hline Iteration & $P$ & $\gamma$ & $\lambda$ & Infeasibility & Objective & $\begin{array}{l}\text { Function } \\
\text { evaluations }\end{array}$ & $\begin{array}{l}\text { Gradient } \\
\text { evaluations }\end{array}$ \\
\hline 1 & 1 & 0 & 0.8119 & 29.64 & 0.8119 & 725 & 151 \\
2 & 1 & $10^{-4}$ & 0.8203 & 9.861 & 0.8193 & 891 & 175 \\
3 & 1 & $10^{-3}$ & 0.8217 & 4.043 & 0.8177 & 541 & 141 \\
4 & 1 & $10^{-2}$ & 0.8168 & 2.754 & 0.7893 & 189 & 60 \\
\hline
\end{tabular}

Table 9: The convergence history of the wing-box results. Note that the infeasibility is calculated as $\left\|\mathbf{c}_{s}\left(\mathbf{x}_{n}^{*}\right)-\mathbf{e}\right\|_{1}$.

As before, we solve a sequence of optimization problems, WingBoxOpt $\left(\gamma_{n}\right)$, with an increasing sequence of penalty parameters, $\gamma_{n}$. We solve the optimization Problem 35, in parallel on 64 processors using TACS [29]. The entire solution requires 2346 function evaluations, 527 gradient evaluations, and just over 65 hours of wall time. Table 9 lists the parameters $\gamma$ and the continuation history of the optimization. The problem does not converge completely to a $0-1$ solution with the present sequence of penalty parameters. Figure 18 shows the lamination sequence results. This figure shows the top half of the lamination sequence. Note that the changes in skin thickness illustrated in Figure 16 are obtained by removing plies symmetrically from the top and bottom of the laminate. The top stiffener and skin plies consist predominantly of $0^{\circ}$ plies. The $15 \%$ ply content constraint on the $90^{\circ}$ plies is active and these plies are placed in the outer-portion of the skin and stiffener laminates. The skin laminate contains many layers of $\pm 45^{\circ}$ plies close to the center of the laminate. Note that four plies in the top skin and stiffener are not converged. Each of these plies consist of a combination of $0^{\circ}$ and $\pm 45^{\circ}$ plies. The bottom skin and stiffener, on the other hand, are composed of a more even mixture of $0^{\circ}, \pm 45^{\circ}$, and $90^{\circ}$ ply angles when compared to the top skin and stiffener. The bottom skin and stiffener, however, contain a total of 5 plies that have not fully converged. Despite the fact that several plies have not fully converged, these solutions could be used as the starting point for further design studies. These results illustrate that the proposed laminate parametrization can be used successfully for a large, complex wing-box design problem.

\section{Conclusions}

In this paper, we have presented a novel laminate parametrization technique that can be used to determine the laminate stacking sequence of a layered composite structure. In this approach, the stiffness and failure criterion are expressed in terms of discrete ply-identity variables. Instead of using discrete variables in the optimization problem, which leads to a nonlinear mixed-integer formulation, we use a continuous relaxation of the discrete problem and impose additional spherical constraints so that the solution to the continuous problem must be a discrete, $0-1$ solution. Instead of introducing these constraints directly into the problem, we add them through an exact $\ell_{1}$-penalty function so that solutions to the relaxed problem are also solutions to the penalized problem, for sufficiently large values 
of the penalty parameter $\gamma$. Additional simplifications can be achieved if the set of linear constraints on the plyidentity variables are satisfied exactly at every iteration in the optimization problem. This approach can be used as an independent penalization, or as an additional penalization to discrete material optimization (DMO) parametrizations that use a SIMP-approach.

We have also presented a technique for discrete thickness selection that accounts for the discrete nature of the thickness of either composite laminates or isotropic materials. In this paper, we have applied these parametrization techniques to a series of structural optimization problems of increasing complexity, including a wing-box sizing and layup-determination study. These results clearly demonstrate that these proposed parametrization methods are effective as either an alternative or an enhancement of SIMP-type approaches. Furthermore, these results demonstrate that the proposed parametrization methods can be applied effectively to a wide range of structural optimization problems.

\section{Acknowledgement}

The computations for the wing-box study presented within this paper were performed on the GPC supercomputer at the SciNet HPC Consortium at the University of Toronto. SciNet is funded by the Canada Foundation for Innovation, under the auspices of Compute Canada, the Government of Ontario, and the University of Toronto.

\section{References}

[1] Tsai, S. W. and Pagano, N. J., "Invariant properties of composite materials," Composite materials workshop, Vol. 1 of Progress in material science, edited by S. W. Tsai, Technomic Publishing Co., 1968, pp. 233-253.

[2] Abrate, S., "Optimal design of laminated plates and shells," Composite Structures, Vol. 29, No. 3, 1994, pp. 269 - 286. doi:10.1016/0263-8223(94)90024-8.

[3] Hammer, V. B., Bendse, M. P., Lipton, R., and Pedersen, P., "Parametrization in laminate design for optimal compliance," International Journal of Solids and Structures, Vol. 34, No. 4, 1997, pp. 415 - 434. doi:10.1016/S0020-7683(96)00023-6.

[4] Fukunaga, H. and Sekine, H., "Stiffness design method of symmetric laminates using lamination parameters," AIAA Journal, Vol. 30, No. 11, 1991, pp. 2791-2793.

[5] Miki, M. and Sugiyama, Y., "Optimum design of laminated composites plates using lamination parameters," AIAA Journal, Vol. 31, No. 5, 1993, pp. 921-922.

[6] Liu, B., Haftka, R., and Trompette, P., "Maximization of buckling loads of composite panels using flexural lamination parameters," Structural and Multidisciplinary Optimization, Vol. 26, 2004, pp. 28-36. doi:10.1007/s00158-003-0314-7.

[7] Foldager, J., Hansen, J. S., and Olhoff, N., "A general approach forcing convexity of ply angle optimization in composite laminates," Structural and Multidisciplinary Optimization, Vol. 16, 1998, pp. 201-211. doi:10.1007/BF01202831.

[8] Jones, R. M., Mechanics of Composite Materials, Technomic Publishing Co., 1996.

[9] IJsselmuiden, S. T., Abdalla, M. M., and Gurdal, Z., "Implementation of strength-based failure criteria in the lamination parameter design space," AIAA Journal, Vol. 46, No. 7, July 2008, pp. 1826-1834. doi:10.2514/1.35565.

[10] Stegmann, J. and Lund, E., "Discrete material optimization of general composite shell structures," International Journal for Numerical Methods in Engineering, 2005, pp. 2009-2027. doi:10.1002/nme.1259.

[11] Bruyneel, M. and Fleury, C., "Composite structures optimization using sequential convex programming," Advances in Engineering Software, Vol. 33, No. 7-10, 2002, pp. 697 - 711. doi:10.1016/S0965-9978(02)00053-4.

[12] Bruyneel, M., "A general and effective approach for the optimal design of fiber reinforced composite structures," Composites Science and Technology, Vol. 66, No. 10, 2006, pp. 1303 - 1314. doi:10.1016/j.compscitech.2005.10.011.

[13] Liu, B., Haftka, R., and Akgun, M., "Two-level composite wing structural optimization using response surfaces," Structural and Multidisciplinary Optimization, Vol. 20, 2000, pp. 87-96. doi:10.1007/s001580050140.

[14] Sigmund, O. and Torquato, S., "Design of materials with extreme thermal expansion using a three-phase topology optimization method," Journal of the Mechanics and Physics of Solids, Vol. 45, No. 6, 1997, pp. 1037 - 1067. doi:10.1016/S00225096(96)00114-7.

[15] Lund, E., "Buckling topology optimization of laminated multi-material composite shell structures," Composite Structures, Vol. 91, No. 2, 2009, pp. 158 - 167. doi:10.1016/j.compstruct.2009.04.046.

[16] Hvejsel, C., Lund, E., and Stolpe, M., "Optimization strategies for discrete multi-material stiffness optimization," Structural and Multidisciplinary Optimization, Vol. 44, 2011, pp. 149-163. doi:10.1007/s00158-011-0648-5.

[17] Beltempo, C. A., Hansen, J. S., and Kennedy, G. J., “Cost-Based Integrated Design Optimization of a Stiffened Plate,” 12th AIAA/ISSMO Multidisciplinary Analysis and Optimization Conference, Victoria, BC, AIAA, 2008.

[18] Haftka, R. T. and Walsh, J. L., "Stacking-sequence optimization for buckling of laminated plates by integer programming," AIAA Journal, Vol. 30, No. 3, March 1992, pp. 814-819. 
[19] Stolpe, M. and Svanberg, K., "On the trajectories of penalization methods for topology optimization," Structural and Multidisciplinary Optimization, Vol. 21, 2001, pp. 128-139. doi:10.1007/s001580050177.

[20] Stolpe, M. and Svanberg, K., "On the trajectories of the epsilon-relaxation approach for stress-constrained truss topology optimization," Structural and Multidisciplinary Optimization, Vol. 21, 2001, pp. 140-151. doi:10.1007/s001580050178.

[21] James, K. A., Hansen, J. S., and Martins, J. R. R. A., "Structural topology optimization for multiple load cases using a dynamic aggregation technique," Engineering Optimization, Vol. 41, No. 12, December 2009, pp. $1103-1118$. doi:10.1080/03052150902926827.

[22] James, K. A., Hansen, J. S., and Martins, J. R. R. A., "Structural topology optimization for multiple load cases while avoiding local minima," April 2008, AIAA 2008-2287.

[23] Nocedal, J. and Wright, S. J., Numerical Optimization, Springer, 1999.

[24] Gill, P. E., Murray, W., and Saunders, M. A., "SNOPT: An SQP Algorithm for Large-Scale Constrained Optimization,” SIAM Review, Vol. 47, No. 1, 2005, pp. pp. 99-131.

[25] Perez, R., Jansen, P., and Martins, J., "pyOpt: A Python-based object-oriented framework for nonlinear constrained optimization,” Structural and Multidisciplinary Optimization, 2011, pp. 1-18. doi:10.1007/s00158-011-0666-3.

[26] Le, C., Norato, J., Bruns, T., Ha, C., and Tortorelli, D., "Stress-based topology optimization for continua," Structural and Multidisciplinary Optimization, Vol. 41, 2010, pp. 605-620. doi:10.1007/s00158-009-0440-y.

[27] Scheel, H. and Scholtes, S., "Mathematical Programs with Complementarity Constraints: Stationarity, Optimality, and Sensitivity," Mathematics of Operations Research, Vol. 25, No. 1, 2000, pp. pp. 1-22.

[28] Scholtes, S., "Convergence Properties of a Regularization Scheme for Mathematical Programs with Complementarity Constraints,” SIAM Journal on Optimization, Vol. 11, No. 4, 2001, pp. 918-936. doi:10.1137/S1052623499361233.

[29] Kennedy, G. J. and Martins, J. R. R. A., "Parallel solution methods for aerostructural analysis and design optimization," Proceedings of the 13th AIAA/ISSMO Multidisciplinary Analysis Optimization Conference, Fort Worth, TX, September 2010, AIAA 2010-9308.

[30] Wrenn, G., “An indirect method for numerical optimization using the Kreisselmeier-Steinhauser function,” NASA Technical Report CR-4220, 1989.

[31] Olhoff, N., "Multicriterion structural optimization via bound formulation and mathematical programming," Structural and Multidisciplinary Optimization, Vol. 1, 1989, pp. 11-17. doi:10.1007/BF01743805.

[32] Seyranian, A. P., Lund, E., and Olhoff, N., "Multiple eigenvalues in structural optimization problems," Structural and Multidisciplinary Optimization, Vol. 8, 1994, pp. 207-227. doi:10.1007/BF01742705.

[33] Liu, B. and Haftka, R., "Single-level composite wing optimization based on flexural lamination parameters," Structural and Multidisciplinary Optimization, Vol. 26, 2004, pp. 111-120. doi:10.1007/s00158-003-0315-6.

[34] Brown, S., "Displacement extrapolation for CFD+CSM aeroelastic analysis," AIAA Paper 97-1090, 1997.

[35] Plank, R. J. and Wittrick, W. H., "Buckling under combined loading of thin, flat-walled structures by a complex finite strip method," International Journal for Numerical Methods in Engineering, Vol. 8, No. 2, 1974, pp. 323-339. doi:10.1002/nme.1620080211.

[36] Akhras, G., Cheung, M., and Li, W., "Static and vibration analysis of anisotropic composite laminates by finite strip method," International Journal of Solids and Structures, Vol. 30, No. 22, 1993, pp. 3129 - 3137. doi:10.1016/0020-7683(93)90143-U.

[37] Stroud, W. J. and Agranoff, N., "Minimum mass design of filamentary composite panels under combined loads: Design procedure based on simplified buckling equations," Tech. rep., NASA Langley Research Center, Hampton, VA 23665, October 1976. 


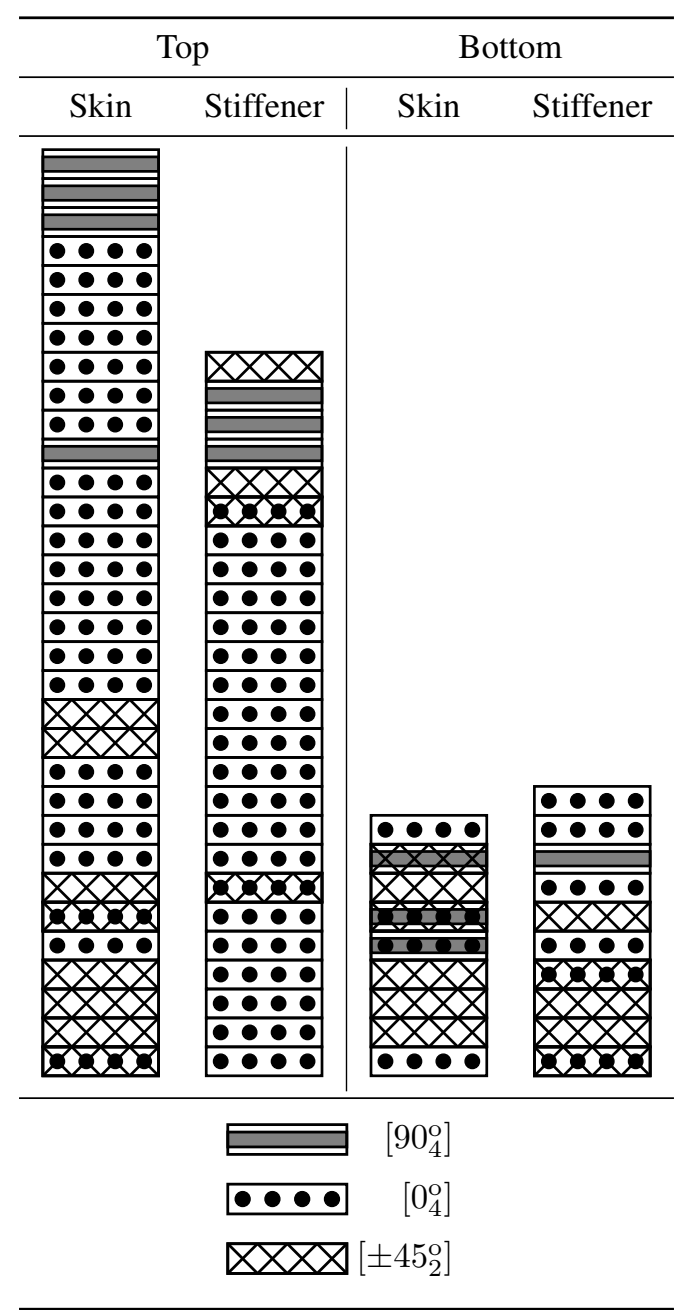

Figure 18: Ply angle sequences for the wing-box optimization problem. Only the top half of the symmetric laminate is shown. Note that not all layers have converged to a $0-1$ point. 